Publ. Mat. 58 (2014), 415-443

DOI: 10.5565/PUBLMAT_58214_21

\title{
ON RESTRICTED WEAK-TYPE CONSTANTS OF FOURIER MULTIPLIERS
}

\author{
AdAM OSȨKOWSKI
}

\begin{abstract}
We exhibit a large class of symbols $m: \mathbb{R}^{d} \rightarrow \mathbb{C}$ for which the corresponding Fourier multipliers $T_{m}$ satisfy the following restricted weak-type estimates: if $A \subset \mathbb{R}^{d}$ has finite Lebesgue measure, then

$$
\left\|T_{m} \chi_{A}\right\|_{p, \infty} \leq \frac{p}{2} e^{(2-p) / p}\left\|\chi_{A}\right\|_{p}, \quad p \geq 2 .
$$

In particular, this leads to novel sharp estimates for the real and imaginary part of the Beurling-Ahlfors operator on $\mathbb{C}$. The proof rests on probabilistic methods: we exploit a stochastic representation of the multipliers in terms of Lévy processes and appropriate sharp inequalities for differentially subordinated martingales.
\end{abstract}

2010 Mathematics Subject Classification: Primary: 42B15, 60G44; Secondary: $42 \mathrm{~B} 20$.

Key words: Fourier multiplier, singular integral, Beurling-Ahlfors transform, martingale, differential subordination.

\section{Introduction}

A linear (or sublinear) operator $T$ defined on $L^{p}\left(\mathbb{R}^{d}\right)$ and taking values in $L^{p, \infty}\left(\mathbb{R}^{d}\right)$ is said to be of restricted weak type $(p, p)$, if there is a constant $C$ such that for every measurable set $A \subset \mathbb{R}^{d}$ of finite Lebesgue measure $|A|$, we have

$$
\left\|T \chi_{A}\right\|_{L^{p, \infty}\left(\mathbb{R}^{d}\right)} \leq C|| \chi_{A} \|_{L^{p}\left(\mathbb{R}^{d}\right)} .
$$

Here the weak $p$-th norm, $1<p<\infty$, is defined by

$$
\|f\|_{L^{p, \infty}\left(\mathbb{R}^{d}\right)}:=\sup \left\{\frac{1}{|E|^{1-1 / p}} \int_{E}|f(x)| \mathrm{d} x\right\},
$$

where the supremum is taken over all measurable $E \subset \mathbb{R}^{d}$ with $0<$ $|E|<\infty$. In most cases, restricted weak type estimates are easier to obtain than the usual strong type inequalities, since the functions involved are bounded and two-valued instead of arbitrary measurable. On the other hand, a pair of restricted weak type estimates is usually powerful enough to imply strong type estimates on intermediate spaces, by means of standard interpolation arguments (see e.g. Corollary 1.4.21 in [19]). 
The purpose of this paper is to study restricted weak-type inequalities for a certain class of Fourier multipliers, with the particular emphasis on the size of the constants. We will show how such estimates can be deduced from appropriate sharp inequalities for differentially subordinated martingales.

To formulate our main results, we need to introduce the necessary background and notation. Suppose that $d \geq 1$ is a fixed integer. For any bounded measurable function $m: \mathbb{R}^{d} \rightarrow \mathbb{C}$, there is a unique bounded linear operator $T_{m}$ on $L^{2}\left(\mathbb{R}^{d}\right)$, called the Fourier multiplier with the symbol $m$, which is given by the identity $\widehat{T_{m} f}=m \widehat{f}$ on Fourier transforms. By Plancherel's theorem, the norm of $T_{m}$ on $L^{2}\left(\mathbb{R}^{d}\right)$ is equal to $\|m\|_{L^{\infty}\left(\mathbb{R}^{d}\right)}$ and there is a natural problem of characterizing those $m$, for which the corresponding Fourier multiplier extends to a bounded linear operator on $L^{p}\left(\mathbb{R}^{d}\right), 1<p<\infty$. The motivation for this question comes from the analysis of classical examples: the collection of Riesz transforms $\left\{R_{j}\right\}_{j=1}^{d}$ on $\mathbb{R}^{d}$ and the Beurling-Ahlfors operator $\mathbb{B}$ on $\mathbb{C}$. Recall that for any $1 \leq j \leq d$, the Riesz transform $R_{j}$ in $\mathbb{R}^{d}$ is the Fourier multiplier corresponding to the symbol $m(\xi)=-i \xi_{j} /|\xi|$, $\xi \in \mathbb{R}^{d} \backslash\{0\}$. Furthermore, the Beurling-Ahlfors transform is an operator acting on functions on $\mathbb{C} \simeq \mathbb{R}^{2}$, corresponding to the symbol $m(\xi)=\bar{\xi} / \xi, \xi \in \mathbb{C} \backslash\{0\}$. One of the motivations for investigating sharp estimates for these operators comes from the papers of Donaldson and Sullivan [15], and Iwaniec and Martin [20], [21], in which it was pointed out that good estimates for the $L^{p}$ norm of these objects have important consequences in the study of quasiconformal mappings, related nonlinear geometric PDEs as well as in the $L^{p}$-Hodge decomposition theory. This justifies our interest in restricted weak-type bounds and, in particular, in obtaining sharp versions of such results.

We will consider the following class of symbols, introduced by Bañuelos and Bogdan in [4]. Assume that $\nu$ is a Lévy measure on $\mathbb{R}^{d}$, i.e., a nonnegative Borel measure on $\mathbb{R}^{d}$ such that $\nu(\{0\})=0$ and

$$
\int_{\mathbb{R}^{d}} \min \left\{|x|^{2}, 1\right\} \nu(\mathrm{d} x)<\infty .
$$

Assume further that $\mu$ is a finite nonnegative Borel measure on the unit sphere $\mathbb{S}$ of $\mathbb{R}^{d}$ and fix two Borel functions $\phi$ on $\mathbb{R}^{d}$ and $\psi$ on $\mathbb{S}$ which take values in the unit ball of $\mathbb{C}$. We define the associated multiplier $m=$ $m_{\phi, \psi, \mu, \nu}$ on $\mathbb{R}^{d}$ by

$$
m(\xi)=\frac{\frac{1}{2} \int_{\mathbb{S}}\langle\xi, \theta\rangle^{2} \psi(\theta) \mu(\mathrm{d} \theta)+\int_{\mathbb{R}^{d}}[1-\cos \langle\xi, x\rangle] \phi(x) \nu(\mathrm{d} x)}{\frac{1}{2} \int_{\mathbb{S}}\langle\xi, \theta\rangle^{2} \mu(\mathrm{d} \theta)+\int_{\mathbb{R}^{d}}[1-\cos \langle\xi, x\rangle] \nu(\mathrm{d} x)}
$$


if the denominator is not 0 , and $m(\xi)=0$ otherwise. Here $\langle\cdot, \cdot\rangle$ stands for the scalar product in $\mathbb{R}^{d}$. This class contains many interesting examples, we refer the interested reader to $[\mathbf{3}]$ and $[\mathbf{4}]$ for the full exposition. We mention here that for $d=2$, these include the real and imaginary part of the Beurling-Ahlfors operator: $\operatorname{Re} \mathbb{B}=R_{2}^{2}-R_{1}^{2}$ and $\operatorname{Im} \mathbb{B}=-2 R_{1} R_{2}$, where $R_{1}, R_{2}$ are the planar Riesz transforms (i.e., Riesz transforms in $\left.\mathbb{R}^{2}\right)$. See Section 4 below.

The Fourier multipliers corresponding to the above symbols can be given a martingale representation by the use of transformations of jumps of Lévy processes (see [3] and [4]). Combining this representation with Burkholder's martingale inequalities, Bañuelos and Bogdan [4] and Bañuelos, Bielaszewski, and Bogdan [3] obtained the following $L^{p}$ bound.

Theorem 1.1. Let $1<p<\infty$ and let $m=m_{\phi, \psi, \mu, \nu}$ be given by (1.1). Then for any $f \in L^{p}\left(\mathbb{R}^{d}\right)$ we have

$$
\left\|T_{m} f\right\|_{L^{p}\left(\mathbb{R}^{d}\right)} \leq\left(p^{*}-1\right)\|f\|_{L^{p}\left(\mathbb{R}^{d}\right)},
$$

where $p^{*}=\max \{p, p /(p-1)\}$.

We should point out here that the special case of this result, concerning the multipliers $R_{2}^{2}-R_{1}^{2}$ and $-2 R_{1} R_{2}$, was obtained earlier by Volberg and Nazarov in [30]. Consult also [5].

It turns out that the above constant $p^{*}-1$ cannot be replaced by a smaller number, which has been shown recently by Geiss, MontgomerySmith, and Saksman [18] (see also [6]).

In fact, the martingale methods allow to establish other types of estimates for the above class of Fourier multipliers. See e.g. [26] for related weak-type inequalities and [27] for corresponding logarithmic bounds. The purpose of this paper is to continue this line of research and study the corresponding restricted weak-type estimates. By interpolation, these lead to further tight bounds on various intermediate spaces. Here is the main result of the paper.

Theorem 1.2. Suppose $T_{m}$ is the Fourier multiplier corresponding to a symbol from the class (1.1). Then for any $A \subset \mathbb{R}^{d}$ of finite Lebesgue measure and any $p \geq 2$ we have

$$
\left\|T_{m} \chi_{A}\right\|_{L^{p, \infty}\left(\mathbb{R}^{d}\right)} \leq \frac{p}{2} e^{(2-p) / p}\left\|\chi_{A}\right\|_{L^{p}\left(\mathbb{R}^{d}\right)}
$$

and the inequality is sharp.

Here by sharpness we mean that for each $p \geq 2$, each $d \geq 2$, and each $\varepsilon>0$ we can find a Fourier multiplier $T_{m}$ with a symbol of the 
form (1.1) and a subset $A$ of $\mathbb{R}^{d}$ such that

$$
\left\|T_{m} \chi_{A}\right\|_{L^{p, \infty}\left(\mathbb{R}^{d}\right)}>\left(\frac{p}{2} e^{(2-p) / p}-\varepsilon\right)\left\|\chi_{A}\right\|_{L^{p}\left(\mathbb{R}^{d}\right)}
$$

Actually, these extremal multipliers will be of the form $R_{1}^{2}-R_{2}^{2}$; thus, in particular, we will obtain that (1.3) is sharp for the real and imaginary parts of the Beurling-Ahlfors operator (it is easy to show that $2 R_{1} R_{2}$ and $R_{1}^{2}-R_{2}^{2}$ have the same restricted weak-type constants, see Section 4).

Unfortunately, we have been unable to find a sharp version of the above statement for $1<p<2$. To explain the reason for this, we need to say a few words about our approach. Roughly speaking, any inequality for Fourier multipliers with symbols as in (1.1) gives rise to a corresponding estimate for differentially subordinate martingales (see Section 2 for the necessary definitions). There is a beautiful method, invented by Burkholder (see [11], [12], and [25]) for proving probabilistic results of this type: the validity of a given martingale inequality (under differential subordination) is equivalent to the existence of a certain special function, satisfying appropriate majorization and concavity properties. In this paper, we will exploit this technique; however, we have managed to find the special function corresponding to the martingale version of (1.3) only for $p \geq 2$. Nonetheless, the results obtained in [26] allow us to write

$$
\left\|T_{m} \chi_{A}\right\|_{L^{p, \infty}\left(\mathbb{R}^{d}\right)} \leq\left[\frac{1}{2} \Gamma\left(\frac{2 p-1}{p-1}\right)\right]^{(p-1) / p}\left\|\chi_{A}\right\|_{L^{p}\left(\mathbb{R}^{d}\right)}, \quad 1<p<2
$$

where $\Gamma$ denotes Euler's gamma function: in fact, this holds true if we replace $\chi_{A}$ by an arbitrary function $f \in L^{p}\left(\mathbb{R}^{d}\right)$. Unfortunately, the above constant does not seem to be the best possible.

Consider the following application (cf. [28]). Let $T_{m}$ be a Fourier multiplier on $\mathbb{R}^{d}$, with a symbol of the form (1.1). Then for any realvalued function $f \in L^{p, 1}\left(\mathbb{R}^{d}\right), p \geq 2$, we have

$$
\left\|T_{m} f\right\|_{L^{p, \infty}\left(\mathbb{R}^{d}\right)} \leq 2^{-1 / p} e^{(2-p) / 2}\|f\|_{L^{p, 1}\left(\mathbb{R}^{d}\right)} .
$$

To see this, assume first that $f=\sum_{j=1}^{N} a_{j} \chi_{E_{j}}$, where $a_{1}>a_{2}>\cdots>$ $a_{N}>0$ and $E_{j}$ are pairwise disjoint subsets of $\mathbb{R}^{d}$ of finite measure. Let $F_{0}=\emptyset$ and $F_{j}=E_{1} \cup E_{2} \cup \cdots \cup E_{j}, j=1,2, \ldots, N$. Then $f$ can be 
rewritten in the form $f=\sum_{j=1}^{N}\left(a_{j}-a_{j+1}\right) \chi_{F_{j}}$, where $a_{N+1}=0$, and

$$
\begin{aligned}
\left\|T_{m} f\right\|_{L^{p, \infty}\left(\mathbb{R}^{d}\right)} & \leq \sum_{j=1}^{N}\left(a_{j}-a_{j+1}\right)\left\|T_{m} \chi_{F_{j}}\right\|_{L^{p, \infty}\left(\mathbb{R}^{d}\right)} \\
& \leq \frac{p}{2} e^{(2-p) / p} \sum_{j=1}^{N}\left(a_{j}-a_{j+1}\right)\left\|\chi_{F_{j}}\right\|_{p} \\
& =\frac{p}{2} e^{(2-p) / p} \sum_{j=0}^{N-1} a_{j+1}\left(\left|F_{j+1}\right|^{1 / p}-\left|F_{j}\right|^{1 / p}\right) \\
& =\frac{1}{2} e^{(2-p) / p}|| f \|_{L^{p, 1}\left(\mathbb{R}^{d}\right)} .
\end{aligned}
$$

By standard approximation, the above inequality extends to any nonnegative $f \in L^{p, \infty}\left(\mathbb{R}^{d}\right)$. To pass to general real-valued functions, it suffices to use the decomposition $f=f_{+}-f_{-}$and the inequality $\left\|f_{+}\right\|_{L^{p, 1}\left(\mathbb{R}^{d}\right)}+$ $\left\|f_{-}\right\|_{L^{p, 1}\left(\mathbb{R}^{d}\right)} \leq 2^{1-1 / p}\|f\|_{L^{p, 1}\left(\mathbb{R}^{d}\right)}$.

We conclude this section by indicating an important connection between the subject of this paper and the theory of quasiconformal mappings. Recall that a homeomorphism $F: \mathbb{C} \rightarrow \mathbb{C}$ is said to be $K$-quasiconformal, $K \geq 1$, if $F \in W_{\text {loc }}^{1,2}(\mathbb{C}, \mathbb{C})$ and $|\bar{\partial} F(z)| \leq \frac{K-1}{K+1}|\partial F(z)|$ for almost all $z \in \mathbb{C}$. In the fifties, Bojarski $[\mathbf{8}],[\mathbf{9}]$ applied the $L^{p}$-boundedness of the Beurling-Ahlfors operator $\mathbb{B}$ to prove that partial derivatives of $K$-quasiconformal maps, which are a priori locally square integrable, belong in fact to $L_{\text {loc }}^{p}$ for some $p>2$ depending only on $K$. By Hölder's inequality, this stronger integrability yields the distortion of area by quasiconformal maps. Formally, if $F(0)=0$ and $F(1)=1$, then for all measurable subsets $E$ of the unit $\operatorname{disc} D=\{z \in \mathbb{C}:|z|<1\}$ we have

$$
|F(E)| \leq C|E|^{\kappa},
$$

where the constants $C$ and $\kappa$ depend only on $K$. Gehring and Reich conjectured in [17] that the least possible $\kappa$ for which (1.5) holds is equal to $1 / K$. This conjecture was open for about 25 years, and was finally proved to be true by Astala [1] two decades ago. This result is strictly related to the following estimate. The weak-type $(1,1)$ and $L^{2}$ boundedness of the Beurling-Ahlfors operator imply the existence of some universal constants $c$ and $\alpha$ such that for any subset $E$ of the unit disc,

$$
\int_{D}\left|\mathbb{B} \chi_{E}\right| \leq \alpha|E| \log \frac{c}{|E|}
$$


Gehring and Reich [17] proved that their conjecture is strictly related to the optimal value of the constant $\alpha$ : roughly speaking, the conjecture is equivalent to saying that the best $\alpha$ equals 1 . The results of this paper yield the following related statement showing how the size of $E$ controls the integral $\int_{D}\left|\mathbb{B} \chi_{E}\right|$. Since the real and imaginary parts of $\mathbb{B}$ have symbols as in (1.1), inequality (1.3) implies

$$
\int_{D}\left|\mathbb{B} \chi_{E}\right| \leq p e^{(2-p) / p} \pi^{1-1 / p}|E|^{1 / p}, \quad 2 \leq p<\infty .
$$

Similarly, (1.4) gives the following version for $1<p<2$ :

$$
\int_{D}\left|\mathbb{B} \chi_{E}\right| \leq 2^{1 / p} \Gamma\left(\frac{2 p-1}{p-1}\right)^{(p-1) / p}|E|^{1 / p} .
$$

The paper is organized as follows. The next section contains the main probabilistic part of the paper: we study there appropriate sharp inequalities for martingales, the stochastic versions of (1.3). In Section 3 we combine these estimates with Bañuelos-Bogdan representation of Fourier multipliers (1.1) and thus obtain (1.3). The final part is devoted to the optimality of the constant $\frac{p}{2} e^{(2-p) / p}$. This is accomplished by the analysis of the so-called laminates, important family of probability measures on $\mathbb{R}^{2 \times 2}$. A clever combination of these objects with Burkholder's special functions can lead to sharp lower bounds for $R_{1}^{2}-R_{2}^{2}$ and $-2 R_{1} R_{2}$, as was first observed by Boros, Székelyhidi Jr., and Volberg in [10]. We extend the argument from that paper and obtain the sharpness of (1.3) for $d=2$; to handle the higher dimensional case, we apply a certain transference-type argument.

\section{A new martingale inequality}

As mentioned in the introduction, the results of this paper depend heavily on certain inequalities for differentially subordinated martingales. To study them, let us begin with the necessary definitions and notation. Assume that $(\Omega, \mathcal{F}, \mathbb{P})$ is a complete probability space, equipped with $\left(\mathcal{F}_{t}\right)_{t \geq 0}$, a nondecreasing family of sub- $\sigma$-fields of $\mathcal{F}$, such that $\mathcal{F}_{0}$ contains all the events of probability 0 . Let $X, Y$ be two adapted cádlág martingales, i.e., with right-continuous trajectories that have limits from the left. We assume further that $X$ takes values in the interval $[0,1]$, while $Y$ is $\mathbb{R}^{\nu}$-valued; here $\nu \geq 1$ is a given integer. The symbols $[X, X]$ and $[Y, Y]$ stand for the square brackets of $X$ and $Y$, respectively; see e.g. [14] for the definition in the real-valued case, and extend the notion to the vector setting by $[Y, Y]=\sum_{k=1}^{\nu}\left[Y^{k}, Y^{k}\right]$, where 
$Y^{k}$ is the $k$-th coordinate of $Y$. Following Bañuelos and Wang [7] and Wang [31], we say that $Y$ is differentially subordinate to $X$, if the process $\left([X, X]_{t}-[Y, Y]_{t}\right)_{t>0}$ is nonnegative and nondecreasing as a function of $t$. For example, let $f=\left(f_{n}\right)_{n \geq 0}, g=\left(g_{n}\right)_{n \geq 0}$ be a pair of adapted discrete-time martingales and let us treat them as continuous-time processes (via $X_{t}=f_{\lfloor t\rfloor}, Y_{t}=g_{\lfloor t\rfloor}, t \geq 0$ ). Then the above domination amounts to saying that $\left|d g_{n}\right| \leq\left|d f_{n}\right|$ for all $n$, which is the original definition of differential subordination, due to Burkholder [11]. Here $\left(d f_{n}\right)_{n \geq 0},\left(d g_{n}\right)_{n \geq 0}$ stand for the difference sequences of $f$ and $g$, given by $d f_{0}=f_{0}, d f_{n}=f_{n}-f_{n-1}(n \geq 1)$, and similarly for $d g$.

The main result of this section can be stated as follows.

Theorem 2.1. Assume that $X, Y$ are martingales taking values in $[0,1]$ and $\mathbb{R}^{\nu}$, respectively. If $Y$ is differentially subordinate to $X$, then for any $\lambda \geq 1 / 2$ we have

$$
\sup _{t \geq 0} \mathbb{E}\left(\left|Y_{t}\right|-\lambda\right)_{+} \leq \frac{e^{1-2 \lambda}}{2} \mathbb{E} X_{0} .
$$

The inequality is sharp, even in the discrete-time setting: for any $\varepsilon>0$ there is a martingale $f$ taking values in $[0,1]$ and a real-valued martingale $g$ which is differentially subordinate to $f$, satisfying

$$
\sup _{n \geq 0} \mathbb{E}\left(\left|g_{n}\right|-\lambda\right)_{+}>\left(\frac{e^{1-2 \lambda}}{2}-\varepsilon\right) \mathbb{E} f_{0} .
$$

As we have already mentioned in the previous section, the proof is based on Burkholder's method and exploits the properties of a certain special function. To introduce this object, fix $\lambda \geq 1 / 2$ and let us first distinguish the following subsets of the strip $[0,1] \times \mathbb{R}^{\nu}$ :

$$
\begin{aligned}
& D_{0}=\{(x, y):|y| \leq \min \{x, 1-x\}\}, \\
& D_{1}=\{(x, y): 0 \leq x \leq 1 / 2, x<|y|<x+\lambda-1 / 2\}, \\
& D_{2}=\{(x, y): 1 / 2<x \leq 1,1<x+|y|<\lambda+1 / 2\}, \\
& D_{3}=\left([0,1] \times \mathbb{R}^{\nu}\right) \backslash\left(D_{0} \cup D_{1} \cup D_{2}\right) .
\end{aligned}
$$

Now, let $U:[0,1] \times \mathbb{R}^{\nu} \rightarrow \mathbb{R}$ be given by

$$
U(x, y)= \begin{cases}\frac{1}{2} \exp (1-2 \lambda)\left(|y|^{2}-x^{2}+x\right) & \text { if }(x, y) \in D_{0}, \\ \frac{1}{2} x \exp (2|y|-2 x-2 \lambda+1) & \text { if }(x, y) \in D_{1}, \\ \frac{1}{2}(1-x) \exp (2|y|+2 x-2 \lambda-1) & \text { if }(x, y) \in D_{2}, \\ \frac{1}{2}\left[(|y|-\lambda+1 / 2)^{2}-x^{2}+x\right] & \text { if }(x, y) \in D_{3} .\end{cases}
$$


We will also need the following auxiliary function $c:[0,1] \times \mathbb{R}^{\nu} \rightarrow[0, \infty)$ :

$$
c(x, y)= \begin{cases}\exp (1-2 \lambda) & \text { if }(x, y) \in D_{0}, \\ \exp (2|y|-2 x-2 \lambda+1) & \text { if }(x, y) \in D_{1} \\ \exp (2|y|+2 x-2 \lambda-1) & \text { if }(x, y) \in D_{2} \\ 1 & \text { if }(x, y) \in D_{3}\end{cases}
$$

In the lemma below, we study the key properties of $U$. We use the dot $\cdot$ to denote the scalar product in $\mathbb{R}^{\nu}$; in addition, for $k \in \mathbb{R}^{\nu}$, we write

$$
\left\langle k U_{y y}(x, y), k\right\rangle=\sum_{i, j=1}^{\nu} U_{y_{i} y_{j}}(x, y) k_{i} k_{j} .
$$

Lemma 2.2. The function $U$ satisfies the following properties.

(i) It is of class $C^{1}$ in $(0,1) \times \mathbb{R}^{\nu}$. Furthermore, if $(x, y)$ belongs to the interior of one of the sets $D_{i}$, then $U$ is infinitely many times differentiable at $(x, y)$.

(ii) If $(x, y)$ belongs to the interior of one of the sets $D_{i}$, then for any $h \in \mathbb{R}$ and $k \in \mathbb{R}^{\nu}$ we have

$$
U_{x x}(x, y) h^{2}+2 U_{x y}(x, y) h \cdot k+\left\langle k U_{y y}(x, y), k\right\rangle \leq c(x, y)\left(|k|^{2}-h^{2}\right) .
$$

(iii) For any $x \in[0,1]$ and $y \in \mathbb{R}^{\nu}$ we have

$$
U(x, y) \geq(|y|-\lambda)_{+} .
$$

Proof: (i) The property formulated in the second sentence is evident. The property from the first sentence is straightforward and reduces to the tedious verification that the function $U$ and its partial derivatives match appropriately at the common boundaries of the sets $D_{i}$. The details are left to the reader.

(ii) The inequality is obvious for $D_{0}^{o}$, the interior of $D_{0}$ : actually, we get equality here. It is also easy to see the validity of (2.2) in the interior of $D_{3}$. Indeed, on this set we have

$$
2 U(x, y)=|y|^{2}-x^{2}+x-2|y|(\lambda-1 / 2)+(\lambda-1 / 2)^{2} .
$$

If the term $2|y|(\lambda-1 / 2)$ were absent, we would have equality in (2.2); since $\lambda \geq 1 / 2$, the function $(x, y) \mapsto 2|y|(\lambda-1 / 2)$ is convex and hence the desired bound is preserved. Next, we turn to the case when $(x, y) \in$ $D_{1}^{o}$. Then it can be computed that the left-hand side of (2.2) is equal 
to $c(x, y)+I+I I$, where

$$
\begin{gathered}
I=\exp (2|y|-2 x-2 \lambda+1)(2 x-1)\left(\frac{y \cdot k}{|y|}-h\right)^{2}, \\
I I=\exp (2|y|-2 x-2 \lambda+1)(x /|y|-1)\left(|k|^{2}-\frac{(y \cdot k)^{2}}{|y|^{2}}\right) .
\end{gathered}
$$

By the definition of $D_{1}$, we have $x \leq 1 / 2$ and $x \leq|y|$, which implies that both $I$ and $I I$ are nonpositive; thus (2.2) follows. Finally, to show the bound for $D_{2}^{o}$, we observe that $U(x, y)=U(1-x, y)$, so the inequality follows at once from the calculations for $D_{1}^{o}$.

(iii) The conditions (i) and (ii) we have just proved yield the following property of $U$ : if $k \in \mathbb{R}^{\nu}$ is an arbitrary vector of norm not exceeding 1 , then for any $y \in \mathbb{R}^{\nu}$ the function $t \mapsto U(t, y+t k), t \in[0,1]$ is concave. Consider the analogous function for the right-hand side of (2.3), i.e., $t \mapsto(|y+t k|-\lambda)_{+}, t \in[0,1]$. This function is convex, so it is enough to check the majorization for $x \in\{0,1\}$. But $U(x, y)=U(1-x, y)$ for all $x \in[0,1], y \in \mathbb{R}^{\nu}$, so all we need is the validity of (2.3) for $x=0$. If $x=0$ and $|y| \leq \lambda-1 / 2$, then both sides of (2.3) are equal to 0 . On the other hand, if $x=0$ and $|y|>\lambda-1 / 2$, we obtain an inequality which is equivalent to the trivial bound $(|y|-\lambda-1 / 2)^{2} \geq 0$. This completes the proof.

Proof of (2.1): The proof rests on an application of Itô's formula to the function $U$ and the martingales $X, Y$. Since $U$ does not have the necessary smoothness, we need some additional regularization of this function. We split the argument into two parts, for the sake of the clarity.

Step 1. A mollified function: Consider a $C^{\infty}$ function $g: \mathbb{R} \times \mathbb{R}^{\nu} \rightarrow$ $[0, \infty)$, supported on the unit ball and satisfying $\int_{\mathbb{R} \times \mathbb{R}^{\nu}} g=1$. Given $\delta \in(0,1 / 2)$, let $U^{\delta}:[\delta, 1-\delta] \times \mathbb{R}^{\nu} \rightarrow \mathbb{R}$ be given by the convolution

$$
U^{\delta}(x, y)=\int_{[-1,1] \times[-1,1]^{\nu}} U(x+\delta u, y+\delta v) g(u, v) \mathrm{d} u \mathrm{~d} v .
$$

Of course, this function is of class $C^{\infty}$. Furthermore, in a sense, it inherits the property studied in Lemma 2.2(ii). Namely, using the first part of the lemma and integrating by parts we obtain, for $x \in[-\delta, 1-\delta]$ and $y \in \mathbb{R}^{\nu}$, that

$$
U_{x x}^{\delta}(x, y)=\int_{[-1,1] \times[-1,1]^{\nu}} U_{x x}(x+\delta u, y+\delta v) g(u, v) \mathrm{d} u \mathrm{~d} v
$$


with similar identities for $U_{x y}^{\delta}$ and $U_{y y}^{\delta}$. Consequently, $U^{\delta}$ satisfies the inequality (2.2) for $x \in[-\delta, 1-\delta]$ and $y \in \mathbb{R}^{\nu}$, with

$$
c^{\delta}(x, y)=\int_{[-1,1] \times[-1,1]^{\nu}} c(x+\delta u, y+\delta v) g(u, v) \mathrm{d} u \mathrm{~d} v
$$

This has the following further consequence: for any $y \in \mathbb{R}^{\nu}$ and any vector $k \in \mathbb{R}^{\nu}$ of norm not exceeding one,

$$
\text { the function } t \mapsto U^{\delta}(t, y+t k), t \in[\delta, 1-\delta] \text {, is concave. }
$$

Step 2. An application of Itô's formula: Fix $\delta \in(0,1 / 2)$. Take martingales $X, Y$ as in the statement of the theorem. Let us modify slightly these processes so that the pair takes values in the set $\{(x, y): \delta \leq x \leq$ $1-\delta,|y| \geq \delta\}$ (then we will be able to compose the martingales with the function $\left.U^{\delta}\right)$. To accomplish this, put $\tilde{X}=\left(\delta+(1-2 \delta) X_{t}\right)_{t \geq 0}$ and $\tilde{Y}=\left((1-2 \delta) Y_{t}\right)_{t \geq 0}$; clearly, the martingale $\tilde{Y}$ is differentially subordinate to $\tilde{X}$.

Let us recall several well-known fact from stochastic analysis. First, there exist a unique continuous local martingale parts $\tilde{X}^{c}$ of $\tilde{X}$ and $\tilde{Y}^{c}$ or $\tilde{Y}$ satisfying

$$
[\tilde{X}, \tilde{X}]_{t}=\left|\tilde{X}_{0}\right|^{2}+\left[\tilde{X}^{c}, \tilde{X}^{c}\right]_{t}+\sum_{0<s \leq t}\left|\Delta \tilde{X}_{s}\right|^{2}
$$

for all $t \geq 0$, and similarly for $\tilde{Y}$. Here $\Delta \tilde{X}_{s}=\tilde{X}_{s}-\tilde{X}_{s-\text { denotes the }}$ jump of $\tilde{X}$ at time $s$. Furthermore, we have $\left[\tilde{X}^{c}, \tilde{X}^{c}\right]=[\tilde{X}, \tilde{X}]^{c}$, the pathwise continuous part of $[\tilde{X}, \tilde{X}]$. Here is Lemma 1 from $[\mathbf{3 1}]$.

Lemma 2.3. If $X$ and $Y$ are semimartingales, then $Y$ is differentially subordinate to $X$ if and only if $Y^{c}$ is differentially subordinate to $X^{c}$, the inequality $\left|\Delta Y_{t}\right| \leq\left|\Delta X_{t}\right|$ holds for all $t>0$ and $\left|Y_{0}\right| \leq\left|X_{0}\right|$.

We come back to the proof of (2.1). An application of Itô's formula yields

$$
U^{\delta}\left(\tilde{X}_{t}, \tilde{Y}_{t}\right)=U^{\delta}\left(\tilde{X}_{0}, \tilde{Y}_{0}\right)+I_{1}+I_{2} / 2+I_{3}
$$


where

$$
\begin{aligned}
I_{1}= & \int_{0+}^{t} U_{x}^{\delta}\left(\tilde{X}_{s-}, \tilde{Y}_{s-}\right) \mathrm{d} \tilde{X}_{s}+\int_{0+}^{t} U_{y}^{\delta}\left(\tilde{X}_{s-}, \tilde{Y}_{s-}\right) \mathrm{d} \tilde{Y}_{s}, \\
I_{2}= & \int_{0+}^{t} U_{x x}^{\delta}\left(\tilde{X}_{s-}, \tilde{Y}_{s-}\right) \mathrm{d}[\tilde{X}, \tilde{X}]_{s}^{c} \\
& +2 \int_{0+}^{t} U_{x y}^{\delta}\left(\tilde{X}_{s-}, \tilde{Y}_{s-}\right) \mathrm{d}[\tilde{X}, \tilde{Y}]_{s}^{c}+\int_{0+}^{t} U_{y y}^{\delta}\left(\tilde{X}_{s-}, \tilde{Y}_{s-}\right) \mathrm{d}[\tilde{Y}, \tilde{Y}]_{s}^{c}, \\
I_{3}= & \sum_{0<s \leq t}\left[U^{\delta}\left(\tilde{X}_{s}, \tilde{Y}_{s}\right)-U^{\delta}\left(\tilde{X}_{s-}, \tilde{Y}_{s-}\right)\right. \\
& \left.\quad-U_{x}^{\delta}\left(\tilde{X}_{s-}, \tilde{Y}_{s-}\right) \Delta \tilde{X}_{s}-U_{y}^{\delta}\left(\tilde{X}_{s-}, \tilde{Y}_{s-}\right) \Delta \tilde{Y}_{s}\right] .
\end{aligned}
$$

Let us analyze the terms $I_{1}$ through $I_{3}$ separately. Both summands in $I_{1}$ have mean zero, by the property of stochastic integrals. The term $I_{2}$ is nonpositive: let $0 \leq s_{0}<s_{1} \leq t$. For any $j \geq 0$, let $\left(\eta_{i}^{j}\right)_{1 \leq i \leq i_{j}}$ be a sequence of nondecreasing finite stopping times with $\eta_{0}^{j}=s_{0}, \eta_{i_{j}}^{j}=s_{1}$ such that $\lim _{j \rightarrow \infty} \max _{1 \leq i \leq i_{j}-1}\left|\eta_{i+1}^{j}-\eta_{i}^{j}\right|=0$. Keeping $j$ fixed, we apply, for each $i=0,1,2, \ldots, i_{j}$, the inequality (2.2) (or rather its version for $U^{\delta}$ ) to $x=\tilde{X}_{s_{0}-}, y=\tilde{Y}_{s_{0}-}$, and $h=h_{i}^{j}=\tilde{X}_{\eta_{i+1}^{j}}-\tilde{X}_{\eta_{i}^{j}}, k=k_{i}^{j}=$ $\tilde{Y}_{\eta_{i+1}^{j}}-\tilde{Y}_{\eta_{i}^{j}}$. Summing the obtained $i_{j}+1$ inequalities and letting $j \rightarrow \infty$ yields

$$
\begin{aligned}
U_{x x}^{\delta}\left(\tilde{X}_{s_{0}-}, \tilde{Y}_{s_{0}-}\right)[X, X]_{s_{0}}^{s_{1}} & +2 \sum_{m=1}^{\nu} U_{x y_{m}}^{\delta}\left(\tilde{X}_{s_{0}-}, \tilde{Y}_{s_{0}-}\right)\left[X, Y^{m}\right]_{s_{0}}^{s_{1}} \\
& +\sum_{m=1}^{\nu} \sum_{n=1}^{\nu} U_{y_{m} y_{n}}^{\delta}\left(\tilde{X}_{s_{0}-}, \tilde{Y}_{s_{0}-}\right)\left[Y^{m}, Y^{n}\right]_{s_{0}}^{s_{1}} \\
\leq & c^{\delta}\left(\tilde{X}_{s_{0}-}, \tilde{Y}_{s_{0}-}\right)\left([Y, Y]_{s_{0}}^{s_{1}}-[X, Y]_{s_{0}}^{s_{1}}\right)
\end{aligned}
$$

where we have used the notation $[S, T]_{s_{0}}^{s_{1}}=[S, T]_{s_{1}}-[S, T]_{s_{0}}$. By the differential subordination of $\tilde{Y}$ to $\tilde{X}$ and the condition $c^{\delta} \geq 0$, the left-hand side above is nonpositive; hence, approximating $I_{2}$ by discrete sums, we obtain $I_{2} \leq 0$. Finally, each summand appearing in $I_{3}$ is nonpositive: this follows immediately from (2.4) and the inequality $\left|\Delta \tilde{Y}_{s}\right| \leq\left|\Delta \tilde{X}_{s}\right|$, guaranteed by Lemma 2.3. Taking expectation of both sides of (2.5) and using the above properties of $I_{1}, I_{2}$, and $I_{3}$, we get the estimate $\mathbb{E} U^{\delta}\left(\tilde{X}_{t}, \tilde{Y}_{t}\right) \leq \mathbb{E} U^{\delta}\left(\tilde{X}_{0}, \tilde{Y}_{0}\right)$. Now we 
let $\delta \rightarrow 0$ : then $U^{\delta}\left(\tilde{X}_{t}, \tilde{Y}_{t}\right) \rightarrow U^{\delta}\left(X_{t}, Y_{t}\right)$ almost surely. By the differential subordination we have $\left|\tilde{Y}_{0}\right| \leq \tilde{X}_{0} \leq 1$, so $\mathbb{E} U^{\delta}\left(\tilde{X}_{0}, \tilde{Y}_{0}\right) \rightarrow$ $\mathbb{E} U\left(X_{0}, Y_{0}\right)$, in view of Lebesgue's dominated convergence theorem. Furthermore, since $U$ is bounded from below, so is $U^{\delta}$ and thus Fatou's lemma yields the estimate $\mathbb{E} U\left(X_{t}, Y_{t}\right) \leq \mathbb{E} U\left(X_{0}, Y_{0}\right)$. But, by (2.3), we have $U\left(X_{t}, Y_{t}\right) \geq\left(\left|Y_{t}\right|-\lambda\right)_{+}$; furthermore, directly from the formula for $U$ and the bound $\left|Y_{0}\right| \leq X_{0}$ from Lemma 2.3, we see that $U\left(X_{0}, Y_{0}\right) \leq \frac{e^{1-2 \lambda}}{2} X_{0}$. This yields the desired inequality.

Sharpness of (2.1): To prove the optimality of the constant $e^{1-2 \lambda} / 2$, we will construct appropriate discrete-time martingales $f=\left(f_{n}\right)_{n>0}$, $g=\left(g_{n}\right)_{n \geq 0}$ such that $g$ is differentially subordinate to $f$. When $\lambda=1 / 2$, the martingales $f, g$ are very simple: put $f_{0}=\kappa \in(0,1 / 2)$, let $f_{1}$ be a mean- $\kappa$ random variable taking values 0 and 1 , set $f_{1}=f_{2}=f_{3}=\cdots$ and $g=f$. Then both sides of (2.1) are equal:

$$
\mathbb{E}\left(\left|g_{1}\right|-\lambda\right)_{+}=\frac{1}{2} \mathbb{P}\left(g_{1}=1\right)=\frac{\kappa}{2}=\frac{e^{1-2 \lambda}}{2}\|f\|_{1} .
$$

Suppose now that $\lambda>1 / 2$, let $N$ be a large positive integer and introduce the parameter $\delta=(\lambda-1 / 2) /(2 N)$. For a fixed $\kappa \in(0,1 / 2)$, consider a sequence $\left(\xi_{n}\right)_{n=0}^{2 N+2}$ of independent random variables with the following distributions: we have $\xi_{0}=\kappa$ with probability $1 ; \mathbb{P}\left(\xi_{1}=1 / 2-\right.$ $\kappa)=1-\mathbb{P}\left(\xi_{1}=-\kappa\right)=2 \kappa$; for $n=1,2, \ldots, N$, we have

$$
\mathbb{P}\left(\xi_{2 n}=-\delta\right)=1-\mathbb{P}\left(\xi_{2 n}=1 / 2\right)=(1+2 \delta)^{-1}
$$

and

$$
\mathbb{P}\left(\xi_{2 n+1}=\delta\right)=1-\mathbb{P}\left(\xi_{2 n+1}=\delta-1 / 2\right)=1-2 \delta ;
$$

finally, $\mathbb{P}\left(\xi_{2 N+2}=-1 / 2\right)=\mathbb{P}\left(\xi_{2 N+2}=1 / 2\right)$. We easily see that for $n \geq 1$ the variables $\xi_{n}$ have mean zero, so the process $\left(\xi_{0}+\xi_{1}+\cdots+\xi_{n}\right)_{n=0}^{2 N+2}$ is a martingale (with respect to its natural filtration). Introduce the stopping time $\tau=\inf \left\{n: \xi_{0}+\xi_{1}+\cdots+\xi_{n} \in\{0,1\}\right\}$; it is easy to see that $\tau$ is finite almost surely. Then, by Doob's optional sampling theorem, the processes

$f_{n}=\xi_{0}+\xi_{1}+\xi_{2}+\cdots+\xi_{\tau \wedge n}, \quad g_{n}=\xi_{0}+\xi_{1}-\xi_{2}+\xi_{3}-\xi_{4}+\cdots+(-1)^{n+1} \xi_{\tau \wedge n}$, where $0 \leq n \leq 2 N+2$, are also martingales (note that the first term in $g_{n}$ is $\xi_{0}$, not $\left.-\xi_{0}\right)$. To gain some intuition about $(f, g)$, let us look at its evolution. The pair starts from the point $(\kappa, \kappa)$; then it moves to $(0,0)$ or to $(1 / 2,1 / 2)$. If it went to $(0,0)$, it stays there forever; if it jumped to $(1 / 2,1 / 2)$, it moves to $(1,0)$ (and stops), or to $(1 / 2-\delta, 1 / 2+\delta)$. If the latter possibility occurs, then it moves to $(0,2 \delta)$ (and stays there forever) or to $(1 / 2,1 / 2+2 \delta)$. The pattern of the movement is then 
repeated; after $2 N+1$ steps we have two possibilities: either $f$ entered the set $\{0,1\}$ (and then $g$ belongs to the interval $[0, \lambda-1 / 2)$ ) or we have $\left(f_{2 N+1}, g_{2 N+1}\right)=(1 / 2, \lambda)$. If the second possibility takes place, the final move of $(f, g)$ is to jump to $(0, \lambda+1 / 2)$ or to $(1, \lambda-1 / 2)$.

Clearly, the martingale $g$ is differentially subordinate to $f$ : indeed, we have $\left|d g_{n}\right|=\left|d f_{n}\right|$ for $0 \leq n \leq 2 N+2$. Next, we have $\|f\|_{1}=\mathbb{E} f_{0}=\kappa$ and

$$
\mathbb{E}\left(\left|g_{2 N+2}\right|-\lambda\right)_{+}=\mathbb{P}\left(g_{2 N+2}=\lambda+1 / 2\right) / 2=\mathbb{P}(A) / 2,
$$

where $A=\left\{\xi_{1}=1 / 2-\kappa, \xi_{2}=-\delta, \xi_{3}=\delta, \xi_{4}=-\delta, \xi_{5}=\delta, \ldots, \xi_{2 N}=\right.$ $\left.\left.-\delta, \xi_{2 N+1}=\delta, \xi_{2 N+2}=-1 / 2\right)\right\}$. Since $\xi_{1}, \xi_{2}, \ldots, \xi_{2 N+2}$ are independent, we obtain

$$
\mathbb{E}\left(\left|g_{2 N+2}\right|-\lambda\right)_{+}=\frac{\kappa}{2}\left(\frac{1-2 \delta}{1+2 \delta}\right)^{N} .
$$

Now if we let $N \rightarrow \infty$ and recall that $\delta=(\lambda-1 / 2) /(2 N)$, we see that

$$
\frac{\mathbb{E}\left(\left|g_{2 N+2}\right|-\lambda\right)_{+}}{\|f\|_{1}} \rightarrow e^{1-2 \lambda} / 2,
$$

and the sharpness follows.

The final result of this section is a corollary which contains, in a sense, a probabilistic version of the inequality (1.3).

Corollary 2.4. Suppose that $X, Y$ are as in Theorem 2.1. Then for any $E \in \mathcal{F}, t \geq 0$, and $\lambda \geq 1 / 2$ we have

$$
\mathbb{E}\left|Y_{t}\right| 1_{E} \leq \frac{e^{1-2 \lambda}}{2} \mathbb{E} X_{0}+\lambda \mathbb{P}(E) .
$$

Proof: We have $E=E^{+} \cup E^{-}$, where $E^{+}=E \cap\left\{\left|Y_{t}\right| \geq \lambda\right\}$ and $E^{-}=$ $E \cap\left\{\left|Y_{t}\right|<\lambda\right\}$. Observe that by $(2.1)$,

$$
\mathbb{E}\left(\left|Y_{t}\right|-\lambda\right) 1_{E^{+}} \leq \mathbb{E}\left(\left|Y_{t}\right|-\lambda\right)_{+} \leq \frac{e^{1-2 \lambda}}{2} \mathbb{E} X_{0}
$$

and, obviously, $\mathbb{E}\left(\left|Y_{t}\right|-\lambda\right) 1_{E^{-}} \leq 0$. Adding the two inequalities above yields the claim.

\section{Proof of (1.3)}

We start by recalling the martingale representation of the multipliers from the class (1.1). This is described in full detail in [3] and [4], so we shall be brief. Let $m$ be the multiplier as in (1.1), with the corresponding parameters $\phi, \psi, \mu$, and $\nu$. Assume in addition that $\nu\left(\mathbb{R}^{d}\right)$ is finite and nonzero. Then for any $s<0$ there is a Lévy process $\left(X_{s, t}\right)_{t \in[s, 0]}$ with 
$X_{s, s} \equiv 0$, for which Lemmas 3.1 and 3.2 below hold true. To state these, we need some notation. For a given $f \in L^{\infty}\left(\mathbb{R}^{d}\right)$, define the corresponding parabolic extension $\mathcal{U}_{f}$ to $(-\infty, 0] \times \mathbb{R}^{d}$ by

$$
\mathcal{U}_{f}(s, x)=\mathbb{E} f\left(x+X_{s, 0}\right)
$$

Next, fix $x \in \mathbb{R}^{d}, s<0$ and let $f, \phi \in L^{\infty}\left(\mathbb{R}^{d}\right)$. We introduce the processes $F=\left(F_{t}^{x, s, f}\right)_{s \leq t \leq 0}$ and $G=\left(G_{t}^{x, s, f, \phi}\right)_{s \leq t \leq 0}$ by

$$
\begin{aligned}
F_{t}= & \mathcal{U}_{f}\left(t, x+X_{s, t}\right) \\
G_{t}= & \sum_{s<u \leq t}\left[\left(F_{u}-F_{u-}\right) \cdot \phi\left(X_{s, u}-X_{s, u-}\right)\right] \\
& -\int_{s}^{t} \int_{\mathbb{R}^{d}}\left[\mathcal{U}_{f}\left(v, x+X_{s, v-}+z\right)-\mathcal{U}_{f}\left(v, x+X_{s, v-}\right)\right] \phi(z) \nu(\mathrm{d} z) \mathrm{d} v .
\end{aligned}
$$

Now, fix $s<0$ and define the operator $\mathcal{S}=\mathcal{S}^{s, \phi, \nu}$ by the bilinear form

$$
\int_{\mathbb{R}^{d}} \mathcal{S} f(x) g(x) \mathrm{d} x=\int_{\mathbb{R}^{d}} \mathbb{E}\left[G_{0}^{x, s, f, \phi} g\left(x+X_{s, 0}\right)\right] \mathrm{d} x,
$$

where $f, g \in C_{0}^{\infty}\left(\mathbb{R}^{d}\right)$. We have the following facts, proved in $[\mathbf{3}]$ and $[\mathbf{4}]$.

Lemma 3.1. For any fixed $x, s, f, \phi$ as above, the processes $F^{x, s, f}$, $G^{x, s, f, \phi}$ are martingales with respect to $\left(\mathcal{F}_{t}\right)_{s \leq t \leq 0}=\left(\sigma\left(X_{s, t}: s \leq\right.\right.$ $t))_{s \leq t \leq 0}$. Furthermore, if $\|\phi\|_{\infty} \leq 1$, then $G^{x, s, f, \bar{\phi}}$ is differentially subordinate to $F^{x, s, f}$.

Let us stress here that $\phi$, and hence also $G$, are complex valued. The aforementioned representation of Fourier multipliers in terms of Lévy processes is as follows.

Lemma 3.2. Let $1<p<\infty$ and $d \geq 2$. The operator $\mathcal{S}^{s, \phi, \nu}$ is well defined and extends to a bounded operator on $L^{p}\left(\mathbb{R}^{d}\right)$, which can be expressed as a Fourier multiplier with the symbol

$$
\begin{aligned}
& \begin{aligned}
M(\xi) & =M_{s, \phi, \nu}(\xi) \\
& =\left[1-\exp \left(2 s \int_{\mathbb{R}^{d}}(1-\cos \langle\xi, z\rangle) \nu(\mathrm{d} z)\right)\right] \frac{\int_{\mathbb{R}^{d}}(1-\cos \langle\xi, z\rangle) \phi(z) \nu(\mathrm{d} z)}{\int_{\mathbb{R}^{d}}(1-\cos \langle\xi, z\rangle) \nu(\mathrm{d} z)}
\end{aligned} \\
& \text { if } \int_{\mathbb{R}^{d}}(1-\cos \langle\xi, z\rangle) \nu(\mathrm{d} z) \neq 0, \text { and } M(\xi)=0 \text { otherwise. }
\end{aligned}
$$


We are ready to establish the inequality (1.3). We may and do assume that at least one of the measures $\mu, \nu$ is nonzero. It is convenient to split the reasoning into two parts.

Step 1: First we show the estimate for the multipliers of the form

$$
M_{\phi, \nu}(\xi)=\frac{\int_{\mathbb{R}^{d}}(1-\cos \langle\xi, z\rangle) \phi(z) \nu(\mathrm{d} z)}{\int_{\mathbb{R}^{d}}(1-\cos \langle\xi, z\rangle) \nu(\mathrm{d} z)} .
$$

Assume that $0<\nu\left(\mathbb{R}^{d}\right)<\infty$, so that the above machinery using Lévy processes is applicable. Fix $s<0$ and functions $f, g \in C_{0}^{\infty}\left(\mathbb{R}^{d}\right)$ such that $f$ takes values in $[0,1]$, while $g$ takes values in the unit ball of $\mathbb{C}$ and is supported on a certain set $E$ of finite Lebesgue measure. Of course, then the martingale $F^{x, s, f}$ takes values in $[0,1]$. By Fubini's theorem and (2.7), we have, for any $\lambda \geq 1 / 2$,

$$
\begin{aligned}
\left|\int_{\mathbb{R}^{d}} \mathbb{E}\left[G_{0}^{x, s, f, \phi} g\left(x+X_{s, 0}\right)\right] \mathrm{d} x\right| \leq & \int_{\mathbb{R}^{d}} \mathbb{E}\left|G_{0}^{x, s, f, \phi}\right| 1_{\left\{x+X_{s, 0} \in E\right\}} \mathrm{d} x \\
\leq & \frac{e^{1-2 \lambda}}{2} \int_{\mathbb{R}^{d}} \mathbb{E}\left|F_{0}^{x, s, f}\right| \mathrm{d} x \\
& +\lambda \int_{\mathbb{R}^{d}} \mathbb{P}\left(x+X_{s, 0} \in E\right) \mathrm{d} x \\
= & \frac{e^{1-2 \lambda}}{2}\|f\|_{L^{1}\left(\mathbb{R}^{d}\right)}+\lambda|E| .
\end{aligned}
$$

Plugging this into the definition of $\mathcal{S}$ and taking the supremum over all $g$ as above, we obtain

$$
\int_{E}\left|\mathcal{S}^{s, \phi, \nu} f(x)\right| \mathrm{d} x \leq \frac{e^{1-2 \lambda}}{2}\|f\|_{L^{1}\left(\mathbb{R}^{d}\right)}+\lambda|E| .
$$

Now if we let $s \rightarrow-\infty$, then $M_{s, \phi, \nu}$ converges pointwise to the multiplier $M_{\phi, \nu}$ given by (3.3). By Plancherel's theorem, $\mathcal{S}^{s, \phi, \nu} f \rightarrow T_{M_{\phi, \nu}} f$ in $L^{2}\left(\mathbb{R}^{d}\right)$ and hence there is a sequence $\left(s_{n}\right)_{n=1}^{\infty}$ converging to $-\infty$ such that $\lim _{n \rightarrow \infty} \mathcal{S}^{s_{n}, \phi, \nu} f \rightarrow T_{M_{\phi, \nu}} f$ almost everywhere. Thus Fatou's lemma combined with (3.4) yields the bound

$$
\int_{E}\left|T_{M_{\phi, \nu}} f(x)\right| \mathrm{d} x \leq \frac{e^{1-2 \lambda}}{2}\|f\|_{L^{1}\left(\mathbb{R}^{d}\right)}+\lambda|E| .
$$

Now, assume that $|E| \leq\|f\|_{L^{1}\left(\mathbb{R}^{d}\right)}$ and minimize the right-hand side over $\lambda$. A straightforward analysis of the derivative shows that the minimum is attained for $\lambda=\left[1+\log \left(\|f\|_{L^{1}\left(\mathbb{R}^{d}\right)} /|E|\right)\right] / 2$, and we obtain the 
estimate

$$
\begin{aligned}
\int_{E}\left|T_{M_{\phi, \nu}} f(x)\right| \mathrm{d} x & \leq\left(1+\frac{1}{2} \log \frac{\|f\|_{L^{1}\left(\mathbb{R}^{d}\right)}}{|E|}\right)|E| \\
& =\left(|E|^{1 / p}+\frac{|E|^{1 / p}}{2} \log \frac{\|f\|_{L^{1}\left(\mathbb{R}^{d}\right)}}{|E|}\right)|E|^{1 / q} .
\end{aligned}
$$

But the expression in the above parentheses does not exceed $\frac{p}{2} e^{(2-p) / p}\|f\|_{L^{1}\left(\mathbb{R}^{d}\right)}^{1 / p}$, which again can be verified by the straightforward manipulations with the derivative. Consequently, we have proved that

$$
\int_{E}\left|T_{M_{\phi, \nu}} f(x)\right| \mathrm{d} x \leq \frac{p}{2} e^{(2-p) / p}\|f\|_{L^{1}\left(\mathbb{R}^{d}\right)}^{1 / p}|E|^{1 / q} .
$$

On the other hand, if the measure of $E$ is larger than $\|f\|_{L^{1}\left(\mathbb{R}^{d}\right)}$, then we apply Schwarz inequality and the $L^{2}$ bound for $T_{M_{\phi, \nu}}$ to get

$$
\begin{aligned}
\int_{E}\left|T_{M_{\phi, \nu}} f(x)\right| \mathrm{d} x & =\int_{\mathbb{R}^{\nu}}\left|T_{M_{\phi, \nu}} f(x)\right| \chi_{E}(x) \mathrm{d} x \\
& \leq\left(\int_{\mathbb{R}^{\nu}}\left|T_{M_{\phi, \nu}} f(x)\right|^{2} \mathrm{~d} x\right)^{1 / 2}|E|^{1 / 2} \\
& \leq\|f\|_{L^{2}\left(\mathbb{R}^{d}\right)}|E|^{1 / 2} \leq\|f\|_{L^{1}\left(\mathbb{R}^{d}\right)}|E|^{1 / 2} .
\end{aligned}
$$

But this is not larger than $\frac{p}{2} e^{(2-p) / p}\|f\|_{L^{1}\left(\mathbb{R}^{d}\right)}^{1 / p}|E|^{1 / q}$, i.e., (3.5) holds true; to prove this bound, rewrite it in the form

$$
\|f\|_{L^{1}\left(\mathbb{R}^{d}\right)}^{1 / 2-1 / p} \leq \frac{p}{2} e^{(2-p) / p}|E|^{1 / q-1 / 2}
$$

and note that $\|f\|_{L^{1}\left(\mathbb{R}^{d}\right)}^{1 / 2-1 / p} \leq|E|^{1 / 2-1 / p}=|E|^{1 / q-1 / 2}$ and $\frac{p}{2} e^{(2-p) / p} \geq$ $\frac{p}{2} \cdot \frac{2}{p}=1$.

Finally, using some standard approximation arguments, we see that (3.5) can be applied to $f=\chi_{A}$ (where $A$ is a measurable subset of $\mathbb{R}^{d}$, satisfying $|A|<\infty)$, and we obtain

$$
\left\|T_{M_{\phi, \nu}} \chi_{A}\right\|_{L^{p, \infty}\left(\mathbb{R}^{d}\right)} \leq \frac{p}{2} e^{(2-p) / p}\left\|\chi_{A}\right\|_{L^{p}\left(\mathbb{R}^{d}\right)} .
$$

Step 2: Now we deduce the result for the general multipliers as in (1.1) and drop the assumption $0<\nu\left(\mathbb{R}^{d}\right)<\infty$. For a given $\varepsilon>0$, define a Lévy measure $\nu_{\varepsilon}$ in polar coordinates $(r, \theta) \in(0, \infty) \times \mathbb{S}$ by

$$
\nu_{\varepsilon}(\mathrm{d} r \mathrm{~d} \theta)=\varepsilon^{-2} \delta_{\varepsilon}(\mathrm{d} r) \mu(\mathrm{d} \theta) .
$$


Here $\delta_{\varepsilon}$ denotes Dirac measure on $\{\varepsilon\}$. Next, consider a multiplier $M_{\varepsilon, \phi, \psi, \mu, \nu}$ as in (3.3), in which the Lévy measure is $1_{\{|x|>\varepsilon\}} \nu+\nu_{\varepsilon}$ and the jump modulator is given by $1_{\{|x|>\varepsilon\}} \phi(x)+1_{\{|x|=\varepsilon\}} \psi(x /|x|)$. Note that this Lévy measure is finite and nonzero, at least for sufficiently small $\varepsilon$. If we let $\varepsilon \rightarrow 0$, we see that

$$
\begin{array}{r}
\int_{\mathbb{R}^{d}}[1-\cos \langle\xi, x\rangle] \psi(x /|x|) \nu_{\varepsilon}(\mathrm{d} x)=\int_{\mathbb{S}}\langle\xi, \theta\rangle^{2} \phi(\theta) \frac{1-\cos \langle\xi, \varepsilon \theta\rangle}{\langle\xi, \varepsilon \theta\rangle^{2}} \mu(d \theta) \\
\rightarrow \frac{1}{2} \int_{\mathbb{S}}\langle\xi, \theta\rangle^{2} \phi(\theta) \mu(\mathrm{d} \theta)
\end{array}
$$

and, consequently, $M_{\varepsilon, \phi, \psi, \mu, \nu} \rightarrow m_{\phi, \psi, \mu, \nu}$ pointwise. Thus (3.6) yields (1.3). Indeed, using Plancherel's theorem as above, we see that there is a sequence $\left(\varepsilon_{n}\right)_{n \geq 1}$ converging to 0 such that $T_{M_{\varepsilon_{n}, \phi, \psi, \mu, \nu}} \chi_{A} \rightarrow T_{m_{\phi, \psi, \mu, \nu}} \chi_{A}$ almost everywhere. It suffices to apply Fatou's lemma.

This completes the proof of the desired bound.

In the remainder of this section we will show how to extend (1.3) to the vector-valued setting. For any bounded function $m=\left(m_{1}, m_{2}, \ldots, m_{n}\right)$ : $\mathbb{R}^{d} \rightarrow \mathbb{C}^{n}$, we may define the associated Fourier multiplier acting on complex valued functions on $\mathbb{R}^{d}$ by the formula $T_{m} f=\left(T_{m_{1}} f, T_{m_{2}} f, \ldots, T_{m_{n}} f\right)$. The above reasoning can be easily modified to yield the following statement.

Theorem 3.3. Let $\nu, \mu$ be two measures on $\mathbb{R}^{d}$ and $\mathbb{S}$, respectively, satisfying the assumptions of Theorem 1.2. Assume further that $\phi, \psi$ are two Borel functions on $\mathbb{R}^{d}$ taking values in the unit ball of $\mathbb{C}^{n}$ and let $m: \mathbb{R}^{d} \rightarrow \mathbb{C}^{n}$ be the associated symbol given by (1.1). Then for any $A \subset \mathbb{R}^{d}$ of finite Lebesgue measure and any $p \geq 2$ we have

$$
\left\|T_{m} \chi_{A}\right\|_{L^{p, \infty}\left(\mathbb{R}^{d} ; \mathbb{C}^{n}\right)} \leq \frac{p}{2} e^{(2-p) / p}\left\|\chi_{A}\right\|_{L^{p}\left(\mathbb{R}^{d}\right)}
$$

and the inequality is sharp.

Proof: Suppose first that $\nu$ is finite. For a given $C^{\infty}$ function $f: \mathbb{R}^{d} \rightarrow$ $[0,1]$, we introduce martingales $F$ and $G=\left(G^{1}, G^{2}, \ldots, G^{n}\right)$ by the formula (3.1). It is easy to check that $G$ is differentially subordinate to $F$, arguing as in $[\mathbf{3}]$ or $[\mathbf{4}]$. Note that $F$ is $[0,1]$-valued, while $G$ takes values in $\mathbb{C}^{n} \simeq \mathbb{R}^{2 n}$. Applying the representation (3.2) to each coordinate of $G$ separately, we obtain the associated multiplier $\mathcal{S}=\left(\mathcal{S}^{1}, \mathcal{S}^{2}, \ldots, \mathcal{S}^{n}\right)$, where $\mathcal{S}^{j}$ has symbol $M_{s, \phi_{j}, \nu_{j}}$ as in Lemma 3.2. Now we repeat the reasoning leading to (3.4), with a vector valued function $g: \mathbb{R}^{d} \rightarrow \mathbb{C}^{n}$ (the expression $G_{0}^{x, s, f, \phi} g\left(x+X_{s, 0}\right)$ should be replaced with the corresponding 
scalar product). An application of (2.1), or rather (2.7), gives

$$
\int_{E}|\mathcal{S} f(x)| \mathrm{d} x \leq \frac{e^{1-2 \lambda}}{2}\|f\|_{L^{1}\left(\mathbb{R}^{d}\right)}+\lambda|E|,
$$

and letting $s \rightarrow-\infty$ yields the corresponding bound for $\mathcal{T}=\left(\mathcal{T}^{1}, \mathcal{T}^{2}, \ldots\right.$, $\mathcal{T}^{n}$ ), where $\mathcal{T}^{j}$ has symbol $M_{\phi_{j}, \nu_{j}}$ as in (3.3). By standard approximation, we may replace $f$ with $\chi_{A}$, and it suffices to apply the optimization procedure over $\lambda$ and $|E|$ to get the claim for these special multipliers. The passage to general $m$ as in (1.1) is carried over in the same manner as in the scalar case.

\section{Sharpness of (1.3)}

We turn to the final part of the paper in which we will show that the constant $\frac{p}{2} e^{(2-p) / p}$ in (1.3) is the best possible. Specifically, we will prove that for any $d \geq 2$, the inequality

$$
\left\|\left(R_{1}^{2}-R_{2}^{2}\right) \chi_{A}\right\|_{L^{p, \infty}\left(\mathbb{R}^{d}\right)} \leq \frac{p}{2} e^{(2-p) / p}\left\|\chi_{A}\right\|_{L^{p}\left(\mathbb{R}^{d}\right)}
$$

is sharp. The proof will be a combination of various analytic and probabilistic facts, and it is convenient to split this section into a several separate parts.

\subsection{Beurling-Ahlfors operator and its action on the indica-} tor function of a ball. Recall that Beurling-Ahlfors operator can be defined as the Fourier multiplier with the symbol $m(\xi)=(\xi /|\xi|)^{2}$, $\xi \in \mathbb{C} \backslash\{0\}$ (with the standard identification $\mathbb{C} \simeq \mathbb{R}^{2}$ ). Alternatively, $\mathbb{B}$ can be expressed as the singular integral

$$
\mathbb{B} f(z)=- \text { p.v. } \frac{1}{\pi} \int_{\mathbb{C}} \frac{f(w)}{(z-w)^{2}} \mathrm{~d} w, \quad z \in \mathbb{C},
$$

where the integration is with respect to the Lebesgue's measure on the complex plane. This operator plays a fundamental role in the study of quasiconformal mappings, partial differential equations and complex analysis. Its importance lies in the fact that it changes the complex derivative $\bar{\partial}$ into $\partial$. Formally, we have

$$
\mathbb{B}(\bar{\partial} w)=\partial w
$$

for every $w$ in the Sobolev space $W^{1,2}(\mathbb{C}, \mathbb{C})$.

The operator $\mathbb{B}$ can be expressed in terms of second-order planar Riesz transforms. To see this, rewrite its symbol in the form

$$
m(\xi)=\frac{\xi^{2}}{|\xi|^{2}}=\frac{\xi_{1}^{2}-\xi_{2}^{2}}{\xi_{1}^{2}+\xi_{2}^{2}}+i \frac{2 \xi_{1} \xi_{2}}{\xi_{1}^{2}+\xi_{2}^{2}}
$$


and observe that $m_{\operatorname{Re}}(\xi)=\left(\xi_{1}^{2}-\xi_{2}^{2}\right) /\left(\xi_{1}^{2}+\xi_{2}^{2}\right)$ is the symbol of $R_{2}^{2}-R_{1}^{2}$ and $m_{\operatorname{Im}}(\xi)=2 \xi_{1} \xi_{2} /\left(\xi_{1}^{2}+\xi_{2}^{2}\right)$ is the symbol of $-2 R_{1} R_{2}$. An important link with the results of this paper states that these multipliers have symbols belonging to the class (1.1). For instance, the choice $d=2$, $\mu=\delta_{(1,0)}+\delta_{(0,1)}, \psi(1,0)=-1=-\psi(0,1)$, and $\nu=0$ gives $T_{m}=\operatorname{Re} \mathbb{B}$; likewise, $d=2, \mu=\delta_{(1 / \sqrt{2}, 1 / \sqrt{2})}+\delta_{(1 / \sqrt{2},-1 / \sqrt{2})}, \psi(1 / \sqrt{2}, 1 / \sqrt{2})=1=$ $-\psi(1 / \sqrt{2},-1 / \sqrt{2})$, and $\nu=0$ leads to $T_{m}=\operatorname{Im} \mathbb{B}$. Furthermore, note that there is a rotation $r$ such that $m_{\mathrm{Re}} \circ r=m_{\mathrm{Im}}$; consequently, the restricted constants of $\operatorname{Re} \mathbb{B}$ and $\operatorname{Im} \mathbb{B}$ are the same. Therefore, having proved (4.1) for $d=2$, we automatically get that $\frac{p}{2} e^{(2-p) / p}$ is the restricted weak constant for both these operators.

We conclude this subsection by recalling a well known fact concerning the action of the operator $\mathbb{B}$ on indicator function of balls. Throughout this section, $\mathcal{B} \subset \mathbb{C}$ denotes the ball of center 0 and radius 1 . Then we have

$$
\mathbb{B} \chi_{\mathcal{B}}(z)=-\frac{1}{z^{2}} \chi_{\mathbb{C} \backslash \mathcal{B}}(z) .
$$

This follows at once from (4.2), by applying it to the function $w(z)=$ $\bar{z} \chi_{\mathcal{B}}+z^{-1} \chi_{\mathbb{C} \backslash \mathcal{B}}$. It will important to us later that in particular, $\left(R_{2}^{2}-\right.$ $\left.R_{1}^{2}\right) \chi_{\mathcal{B}}$ vanishes on $\mathcal{B}$.

4.2. Laminates: necessary definitions. Assume that $\mathbb{R}^{m \times n}$ denotes the space of all real matrices of dimension $m \times n$ and let $\mathbb{R}_{\mathrm{sym}}^{n \times n}$ be the subclass of $\mathbb{R}^{n \times n}$ which consists of all real symmetric $n \times n$ matrices.

Definition 4.1. A function $f: \mathbb{R}^{m \times n} \rightarrow \mathbb{R}$ is said to be rank-one convex, if $t \mapsto f(A+t B)$ is convex for all $A, B \in \mathbb{R}^{m \times n}$ with rank $B=1$.

Let $\mathcal{P}=\mathcal{P}\left(\mathbb{R}^{m \times n}\right)$ stand for the class of all compactly supported probability measures on $\mathbb{R}^{m \times n}$. For $\nu \in \mathcal{P}$, we denote by $\bar{\nu}=\int_{\mathbb{R}^{m \times n}} X d \nu(X)$ the center of mass or barycenter of $\nu$.

Definition 4.2. We say that a measure $\nu \in \mathcal{P}$ is a laminate (and write $\nu \in \mathcal{L})$, if

$$
f(\bar{\nu}) \leq \int_{\mathbb{R}^{m \times n}} f \mathrm{~d} \nu
$$

for all rank-one convex functions $f$. The set of laminates with barycenter 0 is denoted by $\mathcal{L}_{0}\left(\mathbb{R}^{m \times n}\right)$.

Laminates can be used to obtain lower bounds for solutions of certain PDEs, as was first noticed by D. Faraco in [16]. Furthermore, laminates arise naturally in several applications of convex integration, where can be used to produce interesting counterexamples, see e.g. [2], [13], [23], 
[24], and [29]. We will be particularly interested in the case of $2 \times 2$ symmetric matrices. The important fact is that laminates can be regarded as probability measures that record the distribution of the gradients of smooth maps, see Corollary 4.6 below. Let us briefly explain this; detailed proofs of the statements below can be found for example in [22], [24], and [29].

Definition 4.3. Let $U \subset \mathbb{R}^{2 \times 2}$ be a given set. Then $\mathcal{P} \mathcal{L}(U)$ denotes the class of prelaminates in $U$, i.e., the smallest class of probability measures on $U$ which

(i) contains all measures of the form $\lambda \delta_{A}+(1-\lambda) \delta_{B}$ with $\lambda \in[0,1]$ and satisfying $\operatorname{rank}(A-B)=1$;

(ii) is closed under splitting in the following sense: if $\lambda \delta_{A}+(1-\lambda) \nu$ belongs to $\mathcal{P} \mathcal{L}(U)$ for some $\nu \in \mathcal{P}\left(\mathbb{R}^{2 \times 2}\right)$ and $\mu$ also belongs to $\mathcal{P} \mathcal{L}(U)$ with $\bar{\mu}=A$, then also $\lambda \mu+(1-\lambda) \nu$ belongs to $\mathcal{P} \mathcal{L}(U)$.

It follows immediately from the definition that the class $\mathcal{P} \mathcal{L}(U)$ contains atomic measures only. Also, by a successive application of Jensen's inequality, we have the inclusion $\mathcal{P} \mathcal{L} \subset \mathcal{L}$. Let us state two well-known facts (see [2], [22], [24], and [29]).

Lemma 4.4. Let $\nu=\sum_{i=1}^{N} \lambda_{i} \delta_{A_{i}} \in \mathcal{P} \mathcal{L}\left(\mathbb{R}_{\text {sym }}^{2 \times 2}\right)$ with $\bar{\nu}=0$. Moreover, let $0<r<\frac{1}{2} \min \left|A_{i}-A_{j}\right|$ and $\delta>0$. For any bounded domain $\Omega \subset \mathbb{R}^{2}$ there exists $u \in W_{0}^{2, \infty}(\Omega)$ such that $\|u\|_{C^{1}}<\delta$ and for all $i=1, \ldots, N$

$$
\left|\left\{x \in \Omega:\left|D^{2} u(x)-A_{i}\right|<r\right\}\right|=\lambda_{i}|\Omega| \text {. }
$$

Lemma 4.5. Let $K \subset \mathbb{R}_{\mathrm{sym}}^{2 \times 2}$ be a compact convex set and $\nu \in \mathcal{L}\left(\mathbb{R}_{\mathrm{sym}}^{2 \times 2}\right)$ with supp $\nu \subset K$. For any relatively open set $U \subset \mathbb{R}_{\mathrm{sym}}^{2 \times 2}$ with $K \Subset U$ there exists a sequence $\nu_{j} \in \mathcal{P} \mathcal{L}(U)$ of prelaminates with $\bar{\nu}_{j}=\bar{\nu}$ and $\nu_{j} \stackrel{*}{\rightarrow} \nu$.

Combining these two lemmas and using a simple mollification, we obtain the following statement, proved by Boros, Székelyhidi, Jr., and Volberg [10]. It exhibits the connection between laminates supported on symmetric matrices and second derivatives of functions, and will play a crucial role in our argumentation below.

Corollary 4.6. Let $\nu \in \mathcal{L}_{0}\left(\mathbb{R}_{\mathrm{sym}}^{2 \times 2}\right)$. Then there exists a sequence $u_{j} \in$ $C_{0}^{\infty}(\mathcal{B})$ with uniformly bounded second derivatives, such that

$$
\frac{1}{|\mathcal{B}|} \int_{\mathcal{B}} \phi\left(D^{2} u_{j}(x)\right) \mathrm{d} x \rightarrow \int_{\mathbb{R}_{s y m}^{2 \times 2}} \phi \mathrm{d} \nu
$$

for all continuous $\phi: \mathbb{R}_{\mathrm{sym}}^{2 \times 2} \rightarrow \mathbb{R}$. 
Let us stress here that the corollary works for laminates of barycenter 0 . This will give rise to some small technical difficulties, as "natural" laminates do not have this property; see below.

4.3. Biconvex functions and a special laminate. In the next step in our analysis, we introduce a certain special laminate. To do this, we need some additional notation. A function $\zeta: \mathbb{R} \times \mathbb{R} \rightarrow \mathbb{R}$ is said to be biconvex if for any fixed $z \in \mathbb{R}$, the functions $x \mapsto \zeta(x, z)$ and $y \mapsto \zeta(z, y)$ are convex. Now, for $\lambda \geq 1 / 2$, let $f, g$ be martingales of Section 2 , which exhibit the sharpness of (2.1) (if $\lambda$ is strictly larger than $1 / 2$, then there is a whole family of examples, corresponding to different choices of $\kappa$ and $N$ - these two parameters will be specified later). Consider the $\mathbb{R}^{2}$-valued martingale

$$
(F, G):=\left(\frac{f+g}{2}-\kappa, \frac{f-g}{2}\right) .
$$

We subtract $\kappa$ on the first coordinate to ensure that the pair $(F, G)$ has mean $(0,0)$. This sequence has the following zigzag property: for any $0 \leq n \leq 2 N+1$ we have $F_{n}=F_{n+1}$ with probability 1 or $G_{n}=G_{n+1}$ almost surely; that is, in each step $(F, G)$ moves either vertically, or horizontally. Indeed, this follows directly from the construction that for each $n$ we have $\mathbb{P}\left(d f_{n}=d g_{n}\right)=1$ or $\mathbb{P}\left(d f_{n}=-d g_{n}\right)=1$. This property combines nicely with biconvex functions: if $\zeta$ is such a function, then a successive application of Jensen's inequality gives

$$
\begin{aligned}
\mathbb{E} \zeta\left(F_{2 N+2}, G_{2 N+2}\right) & \geq \mathbb{E} \zeta\left(F_{2 N+1}, G_{2 N+1}\right) \geq \cdots \\
& \geq \mathbb{E} \zeta\left(F_{0}, G_{0}\right)=\zeta(0,0) .
\end{aligned}
$$

Now, the martingale $(F, G)$, or rather the distribution of its terminal variable $\left(F_{2 N+2}, G_{2 N+2}\right)$, gives rise to a probability measure $\nu$ on $\mathbb{R}_{\mathrm{sym}}^{2 \times 2}$ : put

$$
\nu(\operatorname{diag}(x, y))=\mathbb{P}\left(\left(F_{2 N+2}, G_{2 N+2}\right)=(x, y)\right), \quad(x, y) \in \mathbb{R}^{2} .
$$

Here and below, $\operatorname{diag}(x, y)$ denotes the diagonal matrix $\left(\begin{array}{ll}x & 0 \\ 0 & y\end{array}\right)$. The key observation is that $\nu$ is a laminate of barycenter 0 (actually, it can be shown that it is even a prelaminate, but we will not need this). To prove this property, note that if $\psi: \mathbb{R}^{2 \times 2}$ is a rank-one convex, then $(x, y) \mapsto \psi(\operatorname{diag}(x, y))$ is biconvex and thus, by $(4.6)$,

$$
\int_{\mathbb{R}^{2 \times 2}} \psi \mathrm{d} \nu=\mathbb{E} \psi\left(\operatorname{diag}\left(F_{2 N+2}, G_{2 N+2}\right) \geq \psi(\operatorname{diag}(0,0))=\psi(\bar{\nu}) .\right.
$$


Finally, note that $\mathbb{P}\left(F_{2 N+2}+G_{2 N+2} \in\{-\kappa, 1-\kappa\}\right)=\mathbb{P}\left(f_{2 N+2} \in\right.$ $\{0,1\})=1$, and hence the support of $\nu$ is contained in

$$
K=\{\operatorname{diag}(x, y): x+y \in\{-\kappa, 1-\kappa\}\} .
$$

4.4. Sharpness of $(\mathbf{1 . 3})$, the case $\boldsymbol{d}=\mathbf{2}$. For the convenience of the reader, let us sketch the idea behind the arguments below. We start with the application of Corollary 4.6 to the laminate $\nu$ : let $\left(u_{j}\right)_{j \geq 1}$ be the corresponding sequence of smooth functions. As we have just observed above, the support of $\nu$ is contained in $K$ given by (4.7). Since the distribution of $u_{j}$ is close to $\nu$ (in the sense of Corollary 4.6), we expect that $\Delta u_{j}$, essentially, takes only values close to $-\kappa$ or close to $1-\kappa$. Thus, if we define $v_{j}=\Delta u_{j}+\kappa \chi_{\mathcal{B}}$ for $j=1,2, \ldots$, then $v_{j}$ is close to an indicator function of a certain set $A$. Thus, to prove the sharpness of $(4.1)$, one can try to study this estimate with $\chi_{A}$ replaced by $v_{j}$. We will look separately at the action on $R_{1}^{2}-R_{2}^{2}$ on $\Delta u_{j}$ and $\kappa_{\chi_{\mathcal{B}}}$; to handle the Laplacian, we will use the arguments from the previous two subsections, and $\kappa \chi_{\mathcal{B}}$ will be dealt with by the facts presented in Subsection 4.1.

Step 1: We start from the specification of the parameters $N$ and $\kappa$. For a given $p \geq 2$, let $\lambda=(p-1) / 2$ and pick an arbitrary number $M$ smaller than $e^{1-2 \lambda} / 2$ : thus, $M=\frac{1}{2} e^{1-2 \lambda} \cdot \eta$ for some $\eta<1$. Let $\kappa \in(0,1 / 2)$ be arbitrary and choose $N$ so that $\mathbb{E}\left(\left|g_{2 N+2}\right|-\lambda\right)_{+}>M \mathbb{E} f_{0}=M \kappa$. This is possible, in view of the results of Section 2. Furthermore, let $\varepsilon$ be an arbitrary positive number (which will eventually be sent to 0 ). In what follows, we will use the following convention: $C_{1}, C_{2}, C_{3}, \ldots$ will denote constants which depend only on $\kappa$ and $N$.

Step 2: Consider a continuous function $\phi: \mathbb{R}_{\mathrm{sym}}^{2 \times 2} \rightarrow \mathbb{R}$ given by $\phi(\operatorname{diag}(x, y))=|x+y+\kappa|$. By Corollary (4.6), we have

$$
\begin{aligned}
\frac{1}{|\mathcal{B}|} \int_{\mathcal{B}}\left|v_{j}\right| & =\frac{1}{|\mathcal{B}|} \int_{\mathcal{B}} \phi\left(D^{2} u_{j}\right) \stackrel{j \rightarrow \infty}{\longrightarrow} \int_{\mathbb{R}_{s y m}^{2 \times 2}} \phi \mathrm{d} \nu \\
& =\mathbb{E}\left|F_{2 N+2}+G_{2 N+2}+\kappa\right|=\kappa,
\end{aligned}
$$

so for sufficiently large $j$ we have

$$
\frac{1}{|\mathcal{B}|} \int_{\mathcal{B}}\left|v_{j}\right| \leq \kappa(1+\varepsilon) \text {. }
$$

Step 3: Consider a continuous function $\phi: \mathbb{R}_{\mathrm{sym}}^{2 \times 2} \rightarrow[0,1]$, satisfying $\phi(\operatorname{diag}(x, y))=1$ when $|x+y-1+\kappa|>\varepsilon$ and $|x+y+\kappa|>\varepsilon$, and 
$\phi(\operatorname{diag}(x, y))=0$ if $x+y+\kappa \in\{0,1\}$. By Corollary 4.6,

$$
\frac{1}{|\mathcal{B}|} \int_{\mathcal{B}} \phi\left(D^{2} u_{j}\right) \rightarrow \int_{\mathbb{R}_{\mathrm{sym}}^{2 \times 2}} \phi \mathrm{d} \nu=0
$$

since $\mathbb{P}\left(F_{2 N+2}+G_{2 N+2}+\kappa \in\{0,1\}\right)=1$. Consider the sets

$$
A=\left\{x \in \mathcal{B}:\left|\Delta u_{j}(x)-1+\kappa\right| \leq \varepsilon\right\}, \quad \tilde{A}=\left\{x \in \mathcal{B}:\left|\Delta u_{j}(x)+\kappa\right| \leq \varepsilon\right\} .
$$

Then (4.9) implies that

$$
\frac{|\mathcal{B} \backslash(A \cup \tilde{A})|}{|\mathcal{B}|}<\varepsilon \quad \text { for sufficiently large } j .
$$

Step 4: Next, consider a continuous function $\phi: \mathbb{R}_{\mathrm{sym}}^{2 \times 2} \rightarrow[0,1]$, satisfying $\phi(\operatorname{diag}(x, y))=1$ if $x+y+\kappa=1$ and $\phi(\operatorname{diag}(x, y))=0$ if $|x+y+\kappa-1|>\varepsilon$. Then

$$
|A| \geq \int_{\mathcal{B}} \phi\left(D^{2} u_{j}\right) \stackrel{j \rightarrow \infty}{\longrightarrow}|\mathcal{B}| \int_{\mathbb{R}_{\mathrm{sym}}^{2 \times 2}} \phi \mathrm{d} \nu=|\mathcal{B}| \mathbb{P}\left(F_{2 N+2}+G_{2 N+2}=1-\kappa\right),
$$

and the number on the right depends only on $N$ and $\kappa$; thus, for large $j$,

$$
|A| \text { is bounded from below by } C_{1} \text {. }
$$

Consequently, for any $1 \leq q<\infty$ and large $j$,

$$
\begin{aligned}
\left\|v_{j}-\chi_{A}\right\|_{L^{q}\left(\mathbb{R}^{2}\right)}^{q}= & \left\|\Delta u_{j}+\kappa-\chi_{A}\right\|_{L^{q}(\mathcal{B})}^{q} \\
= & \int_{A}\left|\Delta u_{j}+\kappa-\chi_{A}\right|^{q}+\int_{\tilde{A}}\left|\Delta u_{j}+\kappa-\chi_{A}\right|^{q} \\
& +\int_{\mathcal{B} \backslash(A \cup \tilde{A})}\left|\Delta u_{j}+\kappa-\chi_{A}\right|^{q} \\
\leq & \varepsilon^{q}|A|+\varepsilon^{q}|\tilde{A}|+\varepsilon|\mathcal{B}|\left(\sup _{\mathcal{B}}\left|\Delta u_{j}\right|+\kappa\right) .
\end{aligned}
$$

Here in the last passage we have used the definition of $A, \tilde{A}$, and (4.10). Combining this with (4.11) (and the fact that the second-order partial derivatives of $u_{j}$ are uniformly bounded by $C_{2}$, see Corollary 4.6), we get that for sufficiently large $j$,

$$
\left\|v_{j}-\chi_{A}\right\|_{L^{q}\left(\mathbb{R}^{2}\right)}^{q} \leq C_{3} \varepsilon|A| .
$$


In other words, the function $v_{j}$ is close to the indicator function of $A$.

Step 5: Next, consider the function $\phi: \mathbb{R}_{\text {sym }}^{2 \times 2} \rightarrow \mathbb{R}$ given by $\phi(\operatorname{diag}(x, y))=$ $(|x-y|-\lambda)_{+}$. By the choice of $\kappa, N$, and (4.8),

$$
\begin{aligned}
\frac{1}{|\mathcal{B}|} \int_{\mathcal{B}} \phi\left(D^{2} u_{j}\right) \stackrel{j \rightarrow \infty}{\longrightarrow} \int \phi \mathrm{d} \nu & =\mathbb{E}\left(\left|g_{2 N+2}\right|-\lambda\right)_{+} \\
& >M \kappa \\
& \geq \frac{M}{1+\varepsilon} \cdot \frac{1}{|\mathcal{B}|} \int_{\mathcal{B}}\left|v_{j}\right| \\
& \geq \frac{M}{1+\varepsilon} \cdot \frac{1}{|\mathcal{B}|}\left(|A|-\int_{\mathcal{B}}\left|v_{j}-\chi_{A}\right|\right) .
\end{aligned}
$$

Now multiply throughout by $|\mathcal{B}|$ and apply (4.12) with $q=1$; we get that for sufficiently large $j$,

$$
\int_{\mathcal{B}} \phi\left(D^{2} u_{j}\right) \geq \frac{M}{1+\varepsilon}\left(1-C_{3} \varepsilon\right)|A| .
$$

However, observe that

$$
\begin{aligned}
\phi\left(D^{2} u_{j}\right) & =\left(\left|\partial_{11} u_{j}-\partial_{22} u_{j}\right|-\lambda\right)_{+} \\
& =\left(\left|\left(R_{1}^{2}-R_{2}^{2}\right) \Delta u_{j}\right|-\lambda\right)_{+}=\left(\left|\left(R_{1}^{2}-R_{2}^{2}\right) v_{j}\right|-\lambda\right)_{+}
\end{aligned}
$$

on $\mathcal{B}$, since $\left(R_{1}^{2}-R_{2}^{2}\right) \chi_{\mathcal{B}}=0$ on $\mathcal{B}$ (see Subsection 4.1). Therefore, the preceding considerations yield that for large $j$,

$$
\begin{aligned}
\frac{M}{1+\varepsilon}\left(1-C_{3} \varepsilon\right)|A| & \leq \int_{\mathcal{B}}\left(\left|\left(R_{1}^{2}-R_{2}^{2}\right) v_{j}\right|-\lambda\right)_{+} \\
& \leq \int_{\mathcal{B}}\left(\left|\left(R_{1}^{2}-R_{2}^{2}\right) \chi_{A}\right|-\lambda\right)_{+}+\int_{\mathcal{B}}\left|\left(R_{1}^{2}-R_{2}^{2}\right)\left(v_{j}-\chi_{A}\right)\right| .
\end{aligned}
$$

However, the norm of $R_{1}^{2}-R_{2}^{2}$ as an operator on $L^{2}\left(\mathbb{R}^{2}\right)$ is bounded by 1 : see (1.2). Consequently, by Schwarz inequality, (4.12), and then (4.11),

$$
\int_{\mathcal{B}}\left|\left(R_{1}^{2}-R_{2}^{2}\right)\left(v_{j}-\chi_{A}\right)\right| \leq\left\|v_{j}-\left.\chi_{A}\left|\|_{2}\right| \mathcal{B}\right|^{1 / 2} \leq C_{4} \varepsilon^{1 / 2}|A|^{1 / 2} \leq C_{5} \varepsilon^{1 / 2}|A| .\right.
$$

Plugging this into the above inequality, we get that if $j$ is sufficiently large, then

$$
\int_{\mathcal{B}}\left(\left|\left(R_{1}^{2}-R_{2}^{2}\right) \chi_{A}\right|-\lambda\right)_{+} \geq \frac{M}{1+\varepsilon}\left(1-C_{6} \varepsilon^{1 / 2}\right)|A| .
$$


Therefore, if we let $E=\left\{x \in \mathcal{B}:\left|\left(R_{1}^{2}-R_{2}^{2}\right) \chi_{A}\right|>\lambda\right\}$ and recall that $M=\frac{1}{2} e^{1-2 \lambda} \cdot \eta$, then

$$
\begin{aligned}
\int_{E}\left|\left(R_{1}^{2}-R_{2}^{2}\right) \chi_{A}\right| & \geq \frac{M}{1+\varepsilon}\left(1-C_{6} \varepsilon^{1 / 2}\right)|A|+\lambda|E| \\
& \geq \frac{\left(1-C_{4} \varepsilon^{1 / 2}\right) \eta}{1+\varepsilon}\left[\frac{e^{1-2 \lambda}}{2}|A|+\lambda|E|\right] .
\end{aligned}
$$

However, we have $\lambda=(p-1) / 2$. Plugging this above and applying Young inequality, we see that the right-hand side is not smaller than

$$
\frac{\left(1-C_{4} \varepsilon^{1 / 2}\right) \eta}{1+\varepsilon} \cdot \frac{p}{2} e^{(2-p) / p}|| \chi_{A} \|_{L^{p}\left(\mathbb{R}^{2}\right)}|E|^{1-1 / p} .
$$

Using the fact that $\eta<1$ and $\varepsilon>0$ were arbitrary, we obtain that the constant $\frac{p}{2} e^{(2-p) / p}$ is indeed the best possible in (1.3).

4.5. Sharpness of (1.3), the case $d \geq 3$. Suppose that for a fixed $p \geq$ 2 and some positive constant $C$ we have

$$
\int_{E}\left|\left(R_{1}^{2}-R_{2}^{2}\right) \chi_{A}(x)\right| \mathrm{d} x \leq C|| \chi_{A} \|_{L^{p}\left(\mathbb{R}^{d}\right)}|E|^{1-1 / p}
$$

for all measurable subsets $A, E$ of $\mathbb{R}^{d}$ of positive and finite Lebesgue measure. For $t>0$, define the dilation operator $\delta_{t}$ as follows: for any function $g: \mathbb{R}^{2} \times \mathbb{R}^{d-2} \rightarrow \mathbb{R}$, we let $\delta_{t} g(\xi, \zeta)=g(\xi, t \zeta)$; for any $A \subset$ $\mathbb{R}^{2} \times \mathbb{R}^{d-2}$, let $\delta_{t} A=\{(\xi, t \zeta):(\xi, \zeta) \in A\}$. Note that the function $\delta_{t} \chi_{A}$ is supported on $\delta_{t}^{-1} A$ and hence, by (4.13), the operator $T_{t}:=\delta_{t}^{-1} \circ$ $\left(R_{1}^{2}-R_{2}^{2}\right) \circ \delta_{t}$ satisfies

$$
\begin{aligned}
\int_{E}\left|T_{t} \chi_{A}(x)\right| \mathrm{d} x & =t^{d-2} \int_{\delta_{t}^{-1} E}\left|\left(R_{1}^{2}-R_{2}^{2}\right) \circ \delta_{t} \chi_{A}(x)\right| \mathrm{d} x \\
& \leq C\left(t^{d-2} \int_{\delta_{t}^{-1} A}\left|\delta_{t} \chi_{A}(x)\right|^{p} \mathrm{~d} x\right)^{1 / p}\left(t^{d-2}\left|\delta_{t}^{-1} E\right|\right)^{1-1 / p} \\
& =C|| \chi_{A} \|_{L^{p}\left(\mathbb{R}^{d}\right)}|E|^{1-1 / p}
\end{aligned}
$$

It is straightforward to check that the Fourier transform $\mathcal{F}$ satisfies the identity $\mathcal{F}=t^{d-2} \delta_{t} \circ \mathcal{F} \circ \delta_{t}$, so the operator $T_{t}$ enjoys the condition

$$
\widehat{T_{t} \chi_{A}}(\xi, \zeta)=-\frac{\xi_{1}^{2}-\xi_{2}^{2}}{|\xi|^{2}+t^{2}|\zeta|^{2}} \widehat{\chi_{A}}(\xi, \zeta), \quad(\xi, \zeta) \in \mathbb{R}^{2} \times \mathbb{R}^{d-2} .
$$

By Lebesgue's dominated convergence theorem, we have

$$
\lim _{t \rightarrow 0} \widehat{T_{t} \chi_{A}}(\xi, \zeta)=\widehat{T_{0} \chi_{A}}(\xi, \zeta)
$$


in $L^{2}\left(\mathbb{R}^{d}\right)$, where $\widehat{T_{0} \chi_{A}}(\xi, \zeta)=\left(\xi_{2}^{2}-\xi_{1}^{2}\right) \widehat{f}(\xi, \zeta) /|\xi|^{2}$. By Plancherel's theorem and Fatou's lemma, we see that (4.14) implies

$$
\int_{E}\left|T_{0} \chi_{A}(x)\right| \mathrm{d} x \leq C\left\|\chi_{A}\right\|_{L^{p}\left(\mathbb{R}^{d}\right)}|E|^{1-1 / p} .
$$

Now pick an arbitrary set $\tilde{A} \subset \mathbb{R}^{2}$ of non-zero and finite Lebesgue measure and put $A=\tilde{A} \times[0,1]^{d-2}$. Denoting by $\mathcal{R}_{1}$ and $\mathcal{R}_{2}$ the planar Riesz transforms, we see that $T_{0} \chi_{A}(\xi, \zeta)=\left(\mathcal{R}_{1}^{2}-\mathcal{R}_{2}^{2}\right) \chi_{\tilde{A}}(\xi) 1_{[0,1]^{d-2}}(\zeta)$, because of the identity

$$
\widehat{T_{0} \chi_{A}}(\xi, \zeta)=-\frac{\xi_{1}^{2}-\xi_{2}^{2}}{|\xi|^{2}} \widehat{\chi_{\tilde{A}}}(\xi) \widehat{1_{[0,1]^{d-2}}}(\zeta) .
$$

Plug this into (4.15) with the choice $E=\tilde{E} \times[0,1]^{d-2}$, where $\tilde{E}$ is an arbitrary subset of $\mathbb{R}^{2}$ with $0<|\tilde{E}|<\infty$. As the result, we obtain

$$
\int_{\tilde{E}}\left|\left(\mathcal{R}_{1}^{2}-\mathcal{R}_{2}^{2}\right) \chi_{\tilde{A}}(\xi)\right| \mathrm{d} \xi \leq C|| \chi_{\tilde{A}} \|_{L^{p}\left(\mathbb{R}^{2}\right)}|\tilde{E}|^{1-1 / p} .
$$

However, we have shown in the previous subsection that this implies $C \geq p e^{(2-p) / p} / 2$. The proof is complete.

\section{Acknowledgments}

The author would like to express his gratitude to an anonymous referee for the careful reading of the first version of the manuscript and many helpful comments. The research was partially supported by Polish Ministry of Science and Higher Education (MNiSW) grant IP2011 039571 'Iuventus Plus'.

\section{References}

[1] K. Astala, Area distortion of quasiconformal mappings, Acta Math. 173(1) (1994), 37-60. DOI: 10.1007/BF02392568.

[2] K. Astala, D. Faraco, and L. SzÉKelyhidi, JR., Convex integration and the $L^{p}$ theory of elliptic equations, Ann. Sc. Norm. Super. Pisa Cl. Sci. (5) 7(1) (2008), 1-50. DOI : 10.2422/2036-2145. 2008.1.01.

[3] R. Bañuelos, A. Bielaszewski, and K. Bogdan, Fourier multipliers for non-symmetric Lévy processes, in: "Marcinkiewicz centenary volume", Banach Center Publ. 95, Polish Acad. Sci. Inst. Math., Warsaw, 2011, pp. 9-25. DOI: 10.4064/bc95-0-1.

[4] R. Bañuelos And K. Bogdan, Lévy processes and Fourier multipliers, J. Funct. Anal. 250(1) (2007), 197-213. DOI: 10.1016/j. jfa.2007.05.013. 
[5] R. Bañuelos and P. J. Méndez-Hernández, Space-time Brownian motion and the Beurling-Ahlfors transform, Indiana Univ. Math. J. 52(4) (2003), 981-990. DOI: 10.1512/iumj .2003.52.2218.

[6] R. BAÑuelos And A. OsȩKowski, Martingales and sharp bounds for Fourier multipliers, Ann. Acad. Sci. Fenn. Math. 37(1) (2012), 251-263. DOI: 10.5186/aasfm.2012.3710.

[7] R. BAÑuelos ANd G. WANG, Sharp inequalities for martingales with applications to the Beurling-Ahlfors and Riesz transforms, Duke Math. J. 80(3) (1995), 575-600. DOI: 10.1215/S0012-7094-9508020-X.

[8] B. Bojarski, Homeomorphic solutions of Beltrami systems, (Russian), Dokl. Acad. Nauk SSSR 102 (1955), 661-664.

[9] B. V. Boyarskĭ, Generalized solutions of a system of differential equations of first order and of elliptic type with discontinuous coefficients, (Russian), Mat. Sb. N.S. 43(85) (1957), 451-503.

[10] N. Boros, L. SzÉKelyhidi, JR., ANd A. Volberg, Laminates meet Burkholder functions, J. Math. Pures Appl. (9) 100(5) (2013), 687-700. DOI: 10.1016/j.matpur.2013.01.017.

[11] D. L. Burkholder, Boundary value problems and sharp inequalities for martingale transforms, Ann. Probab. 12(3) (1984), 647-702.

[12] D. L. Burkholder, Explorations in martingale theory and its applications, in: "École d'Été de Probabilités de Saint-Flour XIX1989", Lecture Notes in Math. 1464, Springer, Berlin, 1991, pp. 1-66. DOI: 10.1007/BFb0085167.

[13] S. Conti, D. Faraco, and F. Maggi, A new approach to counterexamples to $L^{1}$ estimates: Korn's inequality, geometric rigidity, and regularity for gradients of separately convex functions, Arch. Ration. Mech. Anal. 175(2) (2005), 287-300. DOI: 10.1007/s00205-004-0350-5.

[14] C. Dellacherie and P.-A. Meyer, "Probabilities and potential. B. Theory of martingales", Translated from the French by J. P. Wilson, North-Holland Mathematics Studies 72, North-Holland Publishing Co., Amsterdam, 1982.

[15] S. K. Donaldson And D. P. Sullivan, Quasiconformal 4-manifolds, Acta Math. 163(3-4) (1989), 181-252. DOI: 10.1007/ BF02392736.

[16] D. FARACO, Milton's conjecture on the regularity of solutions to isotropic equations, Ann. Inst. H. Poincaré Anal. Non Linéaire 20(5) (2003), 889-909. DOI: 10.1016/S0294-1449(03) 00014-3.

[17] F. W. Gehring and E. Reich, Area distortion under quasiconformal mappings, Ann. Acad. Sci. Fenn. Ser. A I 388 (1966), 15 pp. 
[18] S. Geiss, S. Montgomery-Smith, and E. Saksman, On singular integral and martingale transforms, Trans. Amer. Math. Soc. 362(2) (2010), 553-575. DOI: 10.1090/S0002-9947-09-04953-8.

[19] L. Grafakos, “Classical Fourier analysis", Second edition, Graduate Texts in Mathematics 249, Springer, New York, 2008.

[20] T. Iwaniec And G. Martin, Quasiregular mappings in even dimensions, Acta Math. 170(1) (1993), 29-81. DOI: 10.1007/ BF02392454.

[21] T. IwaniEC And G. Martin, Riesz transforms and related singular integrals, J. Reine Angew. Math. 473 (1996), 25-57.

[22] B. Kirchheim, Rigidity and geometry of microstructures, Ph.D. Thesis, University of Leipzig (2003), http://www.mis.mpg.de/ publications/other-series/ln/lecturenote-1603.html.

[23] B. Kirchheim, S. Müller, And V. Šverák, Studying nonlinear pde by geometry in matrix space, in: "Geometric analysis and nonlinear partial differential equations", Springer, Berlin, 2003, pp. 347-395.

[24] S. MÜller AND V. ŠverÁK, Convex integration for Lipschitz mappings and counterexamples to regularity, Ann. of Math. (2) 157(3) (2003), 715-742. DOI: 10.4007/annals.2003.157.715.

[25] A. OsȨKOWSKI, "Sharp martingale and semimartingale inequalities", Instytut Matematyczny Polskiej Akademii Nauk. Monografie Matematyczne (New Series) 72, Birkhäuser/Springer Basel AG, Basel, 2012. DOI : 10.1007/978-3-0348-0370-0.

[26] A. OsȨKowski, Logarithmic inequalities for Fourier multipliers, Math. Z. 274(1-2) (2013), 515-530. DOI: 10.1007/s00209-0121083-z.

[27] A. OsȨKowski, Weak-type inequalities for Fourier multipliers with applications to the Beurling-Ahlfors transform, J. Math. Soc. Japan 66(3) (2014), 745-764.

[28] E. M. Stein AND G. Weiss, An extension of a theorem of Marcinkiewicz and some of its applications, J. Math. Mech. 8 (1959), 263-284.

[29] L. SzÉkelyhidi, JR., Counterexamples to elliptic regularity and convex integration, in: "The interaction of analysis and geometry", Contemp. Math. 424, Amer. Math. Soc., Providence, RI, 2007, pp. 227-245. DOI : 10.1090/conm/424/08104.

[30] A. Vol'Berg And F. Nazarov, Heating of the Ahlfors-Beurling operator, and estimates of its norm, (Russian), Algebra $i$ Analiz 15(4) (2003), 142-158; translation in: St. Petersburg Math. J. 15(4) (2004), 563-573. DOI : 10.1090/S1061-0022-04-00822-2. 
[31] G. WANG, Differential subordination and strong differential subordination for continuous-time martingales and related sharp inequalities, Ann. Probab. 23(2) (1995), 522-551.

Department of Mathematics, Informatics and Mechanics University of Warsaw

Banacha 2

02-097 Warsaw

Poland

E-mail address: ados@mimuw.edu.pl

Primera versió rebuda el 22 d'abril de 2013, darrera versió rebuda el 7 de maig de 2013. 